\title{
Beamwidth Optimization and Resource Partitioning Scheme for Localization Assisted mm-wave Communications
}

\author{
${ }^{*}$ Gourab Ghatak ${ }^{1},{ }^{*}$ Remun Koirala ${ }^{2,3,4}$, Antonio De Domenico ${ }^{5}$, Benoît Denis ${ }^{2}$, Davide Dardari ${ }^{4}$, Bernard \\ Uguen $^{3}$, and Marceau Coupechoux ${ }^{6}$ \\ ${ }^{1}$ Indraprastha Institute of Information Technology Delhi (IIIT D), India; ${ }^{2}$ CEA-LETI, Grenoble, France; ${ }^{3}$ University of Rennes 1-IETR \\ (CNRS UMR 6164), Rennes, France; ${ }^{4}$ DEI, University of Bologna, Cesena, Italy; ${ }^{5}$ Huawei R\&D Paris; ${ }^{6}$ LTCI, Telecom Paris, Institut \\ Polytechnique de Paris, France;
}

\begin{abstract}
We study a millimeter wave (mm-wave) wireless network deployed along the roads of an urban area, to support localization and communication services simultaneously for outdoor mobile users. In this network, we propose a mm-wave initial beam-selection scheme based on localization-bounds, which greatly reduces the initial access delay as compared to traditional initial access schemes for standalone mm-wave small cell base station (BS). Then, we introduce a downlink transmission protocol, in which the radio frames are partitioned into three phases, namely, initial access, data, and localization, respectively. We establish a trade-off between the localization and communication performance of $\mathrm{mm}$-wave systems, and show how enhanced localization can actually improve the data-communication performance. Our results suggest that dense BS deployments enable to allocate more resources to the data phase while still maintaining appreciable localization performance. Furthermore, for the case of sparse deployments and large beam dictionary size (i.e., with thinner beams), more resources must be allotted to the localization phase for optimizing the rate coverage. Based on our results, we provide several system design insights and dimensioning rules for the network operators that will deploy the first generation of mm-wave BSs.
\end{abstract}

Index Terms-Millimeter-wave communications, Stochastic Geometry, Localization, Positioning, Initial Access.

\section{INTRODUCTION}

One of the major catalysts for the development of future mobile networks is the increasing demand for high data-rates. Accordingly, due to the large bandwidth available between the $24 \mathrm{GHz}$ and $86 \mathrm{GHz}$ frequency range, mm-wave communication is an integral part of the fifth generation (5G) mobile communication systems [1]. However, transmissions using high frequencies suffer from large attenuation and sensitivity to blockages [2]. Nevertheless, owing to the short wavelength of mm-wave transmissions, antennas are smaller than those in sub- $6 \mathrm{GHz}$ bands. This enables the integration of a larger number of antennas on both transmitter and receiver sides [3]. Consequently, the higher attenuation can be mitigated using directional beamforming techniques [4]. Moreover, the large number of antennas enables the BS to adapt the beam size

\footnotetext{
* These authors have equal contributions in the work.

This work has been carried out in the frame of the SECREDAS project, which is partly funded by the European Commission (H2020 EU.2.1.1.7 ECSEL - GA 783119). The work of M. Coupechoux has been carried out at LINCS (http://www.lincs.fr).
}

in order to optimally trade-off the beamforming gain and the coverage area. Additionally, the usage of directional beamforming greatly reduces co-channel interference [5], which further increases the data-rates.

On the downside, very thin beams raise additional constraints in terms of initial access and coverage [6], which are challenging in the case of standalone deployment of mm-wave BSs [7]. One solution to these challenges in case of a multi-RAT network is to allow the users to simultaneously receive signals in the mm-wave and in the sub- $6 \mathrm{GHz}$ band, and to use the latter to support the initial access to the mm-wave systems [6]. Another approach is first to employ positioning algorithms in order to localize the users with respect to the BSs, and then, to select the proper mm-wave beam to initiate the data transmission [8]. In other words, the position and orientation information of the users relative to the BS can be used as a proxy for channel information to facilitate beamforming. This alleviates the need to undergo an elaborate beam training procedure, which is particularly critical in case of low-latency, high-throughput applications. However, this comes at the cost of an increased error in the configuration of transmit and receive beams due to possible inaccuracies in localization [9]. Thus, to completely harness the potential benefits offered by mm-wave communications, accurate characterization of the spatial configuration (e.g., the relative distance, angle of departure (AoD), and angle of arrival (AoA) between the transmitter and the receiver) is necessary.

We investigate a mm-wave network deployed along the roads of an urban area to support localization and communication services simultaneously. Particularly, we study and optimize a resource partitioning scheme to address jointly localization and communication requirements.

\section{A. Related Work}

The feasibility of providing very high data-rates by operating at mm-wave frequencies is now well established in the literature [4]. Bai et al. [10] and Di Renzo [11] have provided the first works on rate analysis of single-tier and multitier mm-wave communications, respectively, in random urban networks. Furthermore, Elshaer et al. [12] have studied mmwave systems co-existing with traditional sub- $6 \mathrm{GHz}$ infrastructure. However, most of these works either do not consider,

\section{ACCEPTED MANUSCRIPT}


or do not fully address the challenges of providing initial access to the mm-wave terminals. Ghatak et al. [6] have also studied networks with co-existing mm-wave and sub-6GHz radio access techniques (RATs), in which the control signals sent in the sub-6GHz band are used to provide initial access to the mm-wave nodes. However, they have not provided any algorithm for facilitating the initial access procedure. In this direction of research, Li et al. [13] have studied simple initial access protocols involving hybrid directional beamforming and omni-directional transmissions during the cell-search and random access phases. According to their finding, the best trade-off between initial access delay and average downlink throughput is obtained using wide beams in the BS side and beam-sweeping in the user-side. The major concern with such protocols remains the high access delays in case of a high number of beams, especially for systems serving lowlatency applications. Recently, Yang et al. [14] have studied an initial access scheme that substantially reduces the latency with respect to the classical exhaustive and iterative search algorithms.

In the context of user localization, the potential benefits of high-accuracy localization using mm-wave beamforming was relatively unexplored until recently [15]. The initial works in mm-wave localization studied how to derive the CRLB of the location dependent variables (e.g. distance, AoD, and AoA) considering single [16] and multiple carriers [17] using both single [16] and multipath models [17]. Then, the focus was on localization oriented beamforming, considering these theoretical performance bounds. In particular, the authors in [18], [19] studied the localization optimal beamforming problem, considering the joint optimization of the CRLB of the localization variables for both single and multiple user cases. More recently, in [20], the authors presented a beamforming strategy to minimize the localization error expressed in the form of the squared position error bound (SPEB).

From the perspective of joint localization and communication functionalities, Destino et al. [21] and Kumar et al.[22] have studied the trade-off between communication rate and localization quality in a single user and multiuser mm-wave link respectively. Typically, the localization performance is characterized by theoretical bounds that model its accuracy [23], whereas, in order to characterize the communication performance, metrics such as user throughput are derived [13]. Likewise, in [24], the authors present a beam alignment optimization scheme between the transmitter and the receiver considering erroneous position estimations at both ends and scatterers. In this work, the authors describe a 2-step beam alignment algorithm, firstly at the transmitter independently and then at the receiver following the transmitter's decision. Recently in [25], the authors presented a beam alignment method where, under similar conditions as [24], the transmitter and receiver select the beams in a joint manner, thus outperforming the 2-step method. Likewise, in [26], the authors present an iterative localization based beam selection algorithm where the transmitter, in each iteration, selects a refined finer beam based on position and orientation estimation. The refined beam again improves the estimation and the process continues in a virtuous loop. Extending this idea, in [27], the authors present the beam selection algorithm at both transmitter and receiving ends. A work that analyses the trade-off between localization and data communication in random wireless networks appeared as a preliminary conference version of this paper [9], [28]. There, we have used stochastic geometry to derive the signal to interference plus noise ratio (SINR) coverage probability and characterize the data-rate performance during the data service phase given some localization performance during the localization phase. This approach only partially captures the intricate relation between localization and communication performance, precisely because enhancing localization may improve the downlink data-rate in mm-wave systems by reducing beam-selection and misalignment errors.

In this paper, we introduce the notion of effective data-rate that not only takes into account the effect of localization error on the downlink data-rate, but also integrates the overhead due to the initial access scheme. The application of stochastic geometry enables us to formulate the beamwidth optimization and resource-partitioning problem from the perspective of a random user in the network sampled from the distribution of the users. Consequently, the prescribed scheme for optimizing the effective rate-coverage probability of the typical user gives the best expected beamwidth values and the expected resourcepartitioning parameter for the entire network. This enables us to derive essential system design insights for this network. The overall contributions are summarized as follows.

\section{B. Contributions and Organization}

In this paper, we study a mm-wave network with simultaneous localization and communication services in a onedimensional (e.g., along roads) scenario. Particularly, we design and study a downlink transmission scheme where the radio frames are partitioned into initial access, data, and localization phases. In this paper,

1) We recall the FIM and consequently the CRLB for the joint estimation of the distance and AoA of the BSuser link. Based on these, we define and derive two new performance metrics to evaluate the localization performance of the system, namely, the beam selection error and the misalignment error, which are respectively induced by user's position and orientation estimation errors.

2) With the help of the formulated beam selection and misalignment errors, we design the Tx-Rx best beam pair selection strategy for establishing the initial connection between the mm-wave $\mathrm{BS}$ and the user, which reduces the overhead of beam training significantly as compared to the popular beam sweeping methods [13].

3) For the data phase, we provide a more accurate characterization of the downlink SINR coverage probability as compared to the existing studies [9], [28], by taking the errors during the localization phase into account. Leveraging on this mathematical characterization, we highlight the non-trivial localization and data-rate tradeoff in this system. As an example, it may be intuitive to expect that allocating larger amount of resources to the communication phase increases the data-rate. However, 


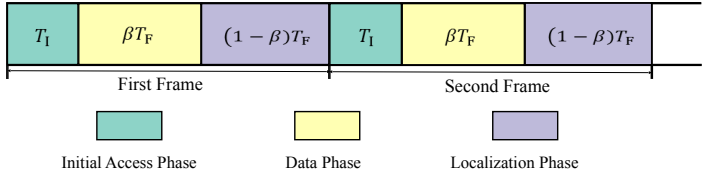

Figure 1. The proposed radio frame structure for localization assisted mmwave communications.

as this results in a shorter localization phase, which leads to less accurate localization of the users, it adversely affects the data-rates. In this work, we optimize this resource partitioning factor (i.e., adapting the resource split between data and localization phases) jointly with the beamwidth of the mm-wave BSs to simultaneously address the localization and communication requirements.

The framework developed in this paper can be used in frame design of an urban mm-wave system, not only for maximization of downlink data-rate, but also to address given data-rate requirements under localization constraints and vice-versa.

The rest of the paper is organized as follows. In Section II, we introduce our system model and outline our optimization objectives. Then, in Section III, we describe the proposed initial access scheme. We provide the performance analysis of the localization and data phases in Section IV, whereas we discuss the overall system performance in Section V. Finally, the paper concludes in Section VI. Table I summarizes the main notations used in this paper.

\section{SYSTEM MODEL}

We consider a small cell network where multi-RAT BSs are deployed along the roads to provide high speed data-access to the mobile users by jointly exploiting sub-6 $\mathrm{GHz}$ and $\mathrm{mm}$-wave bands. In this context, we propose a radio frame structure for joint communication and localization services, which is illustrated in Fig. 1. Each frame consists of an initial access phase of length $T_{I}$ and a service phase of length $T_{F}$. The access phase enables to establish reliable mm-wave services to the new user equipments (UEs) that arrive in the system before the start of that frame. This phase is relevant only for the new UEs and is not repeated for all the UEs. To do so, our approach iteratively increases the resolution of the estimation of the distance and the orientation of the user with respect to the serving BS. The service phase is further partitioned by a factor $\beta$ into a data phase of length $\beta T_{F}$ and a localization phase of length $(1-\beta) T_{F}$. In this paper, we assume that the resources in the initial access and localization phases are perfectly multiplexed across active users, i.e., interference does not affect the localization performance. The service phase is further partitioned by a factor $\beta$ into a data phase of length $\beta T_{F}$ and a localization phase of length $(1-\beta) T_{F}$. The access phase enables to provide reliable mm-wave services to the new UEs in the system. Accordingly, in this phase, the initial beams at the BS and UE sides are refined in an iterative

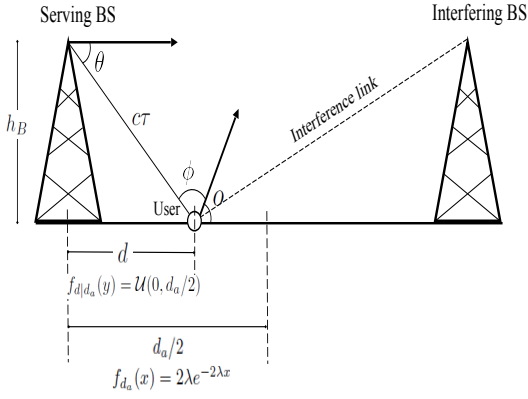

Figure 2. System model consisting of a serving BS, an interfering BS and a user node at distance $d$ from the serving BS. The figure illustrates the relationship between the BS and user positions and the localization variables (distance $d$, AoD $\phi$, AoA $\psi$ and the user orientation $o$ ).

manner, until the localization information (distance and $\mathrm{AoA}^{1}$ ) reaches a predefined resolution. Then, the data and localization phases follow as depicted in Fig. 1. Both downlink and uplink are included in our radio frame structure. Specifically, in the initial access and localization phases there is an exchange of downlink and uplink signals to enable precise estimation of the localization parameters. However, in the following, we analyse the performance of the data-phase exclusively during downlink communications. Initially, the BS selects the transmit beamwidth $\left(\theta^{*}\right)$ to maximize the effective data rate, which takes into account the localization errors as well, and satisfy the localization service requirements. In the following frames, $\theta^{*}$ is further adapted to the obtained position and orientation information in order to improve the system performance. Thus, in the localization phase, the location information of the users are updated. For static users, this information is improved at each subsequent frame. For mobile users, the aim of this phase is to keep a track of the current location so as to facilitate beam-switching if needed. It is important to note that the beamwidth at the BS side that facilitates $\mathrm{mm}$-wave service (in the initial access phase) is different from $\theta^{*}$. The former is refined in an iterative manner in the initial access phase using the algorithm defined in Section III to provide access to new UEs; whereas, the latter is obtained using the framework developed in Section IV.

\section{A. Network Geometry}

Let us consider an urban scenario with multi-storied buildings resulting in a dense blocking environment. The BSs deployed along the roads of the city are assumed to be of height $h_{B}$ and having a transmit power of $P_{t}$. Their positions in each street are modeled as points of a one-dimensional Poisson point process (PPP) $\xi$, with intensity $\lambda\left[\mathrm{m}^{-1}\right]$. The 1D model assumed in this paper is relevant for the case where the cellular deployment is envisioned to be along roads. As an example, Verizon and AT\&T have both announced plans to deploy $5 \mathrm{G}$ infrastructure on lampposts for fixed wireless

\footnotetext{
${ }^{1}$ In this paper, for AoA estimation, we can choose one of the popular techniques such as Bartlett technique [29], Capon technique [30] or a subspace based techniques [31] (Multiple Signal Classification). For distance estimation, one can simply use received signal-strength indicator (RSSI) or time of flight (ToF) based estimation in the mm-wave band [32].
} 
Table I

Main Notations And System Parameters.

\begin{tabular}{|ccc|}
\hline Notation & Parameter & Value \\
\hline \hline$\xi, \lambda$ & BS process and its intensity & $\lambda=5-200 \mathrm{per} \mathrm{km}$. \\
\hline$P_{t}$ & Transmitted power from BS & $30 \mathrm{dBm}$ \\
\hline$\theta_{B / U}$ & Width of the beams of BS/user & - \\
\hline$\theta_{k}$ & Instance of $\theta_{B}$ & - \\
\hline$\psi / \phi$ & AoA/AoD & - \\
\hline$\tau$ & Delay corresponding to LOS distance between BS and the user & - \\
\hline$\beta$ & Resource partitioning factor & 2,4 \\
\hline$\alpha_{L}, \alpha_{N}$ & Approximated LOS/NLOS path-loss exponents \\
\hline$N_{0}$ & Noise power density of the received signal & $-174 \mathrm{dBm} / \mathrm{Hz}$ \\
\hline$B$ & Bandwidth & $1 \mathrm{GHz}$ \\
\hline$\sigma_{d}^{2} / \sigma_{\psi}^{2}$ & Variance of the distance/AoA estimation error & - \\
\hline$d_{S}$ & BS LOS ball radius & $20 \mathrm{~m}$ \\
\hline$f_{c}$ & Carrier Frequency & $60 \mathrm{GHz}$ \\
\hline \hline
\end{tabular}

network [33] and mobile access [34], respectively. In such scenario, the 1D model assumed in our paper can be utilized by a network operator to derive system design insights and to further fine-tune the deployment parameters.

However, it must also be noted that the methodology developed in this paper can be extended to a more general 2D network model, following the models adopted in, e.g., [?]. Then, the coverage of the BSs will be modeled as 2D areas instead of 1D lengths. Accordingly, the notions developed in this paper e.g., beam selection and misalignment errors, and SINR coverage can be extended by using two variables for representing the locations of the users and solid angles to characterize the beam coverage of the small cells.

The users are assumed to be static and located uniformly on the roads with a density $\lambda_{U}\left[\mathrm{~m}^{-1}\right]$. In this regard, it is important to highlight that mobility does not have a large impact on our protocol and performance evaluation methodology. As an example, let us assume vehicular users moving at a speed of 30 $\mathrm{km}$ per hour. With $1 \mathrm{~ms}$ of frame length, the distance covered by the user in-between frames is approximately $8 \mathrm{~cm}$, which is considerably small with respect to the coverage area of any beam in the dictionary. When the user speed is very high or the frame length is large, the effect of the mobility on the user localization can be addressed by increasing the variance of the noise in the estimation of the localization variables.

Without the loss of generality, we perform our analysis from the perspective of a BS located at the origin and an associated user located at a distance $d$ from the BS as illustrated in Fig. 2. The user selects the serving BS following a RSSI based association. For a BS located at the origin, the probability density function (PDF) of its distance from the nearest neighbor (i.e, the closest BS) is given by [35], [36]:

$$
f_{d_{a}}(x)=2 \lambda \exp (-2 \lambda x) .
$$

Then, assuming that all the BSs have equal transmit power, the coverage area of the BS located at the origin is given by $\frac{d_{a}}{2}$ on either side of it, where $d_{a}$ follows the distribution (1). Therefore, inside the coverage region of this BS, the location of a random user is uniformly distributed. Accordingly, the joint probability distribution of the distance $d$ and the coverage area $d_{a}$ is given by $f_{d_{a}, d}(x, y)=f_{d}\left(y \mid d_{a}=x\right) f_{d_{a}}(x)$ [35], where

$$
f_{d}\left(y \mid d_{a}=x\right)=\left\{\begin{array}{l}
x^{-1} ; \quad 0 \leq y \leq x \\
0 ; \quad \text { otherwise }
\end{array} .\right.
$$

In the following, we denote the user orientation with respect to the reference $\mathrm{x}$-axis as $o$, the AoA at the user as $\psi$ and the AoD at the BS as $\phi$. As depicted in Fig. 2, the relation between the position of the BS and the user with the delay $\tau$, AoD, AoA and the user orientation are:

$$
\begin{aligned}
\tau & =\sqrt{d^{2}+h_{B}^{2}} / c, \\
\phi & =\cos ^{-1}\left(d / \sqrt{d^{2}+h_{B}^{2}}\right), \\
\psi & =\pi-\cos ^{-1}\left(d / \sqrt{d^{2}+h_{B}^{2}}\right)-o,
\end{aligned}
$$

where $c$ is the speed of light. It must be noted that in our 1D scenario, $\phi$ is dependent directly on $d$. We also assume that the orientation of the users are unknown, and accordingly, we consider that the distribution of the initial AoA of the user $f(\psi)$ is uniform between 0 and $2 \pi$.

\section{B. Millimeter-Wave Beamforming}

Our analysis consists of two parts, the first one involving derivation of Cramer-Rao lower bound (CRLB) for the localization phase and, then, the derivation of the user performance in the data phase. For the derivation of CRLB for AoA estimation, the angular information is derived from the antenna array response, hence we use the uniform linear array (ULA) model [17] with an antenna spacing of half the carrier wavelength. On the other hand, in order to simplify the analysis of the data phase, we approximate the beamforming by a sectorized model [37], where the transmitted and received beams are divided into two sectors, a main lobe sector whose antenna gain depends on the beamwidth $\theta$ and a side lobe sector with a fixed gain. Here, the term main lobe stands for the angular region of the antenna pattern centered around the axis of maximum gain and aperture equal to the half-power beamwidth of the pattern. We assume that the BSs do not cater to multiple users or transmit multistream data, simultaneously. Accordingly, we assume the existence of a single RF chain with analog beamforming. 


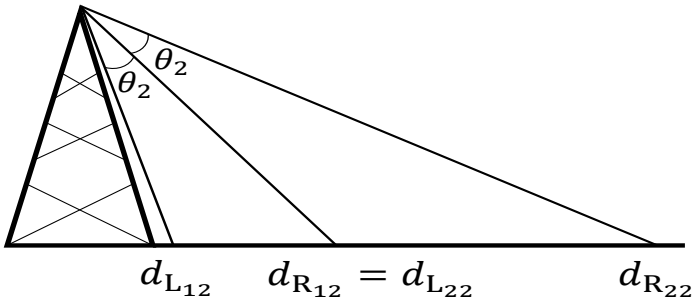

(a)

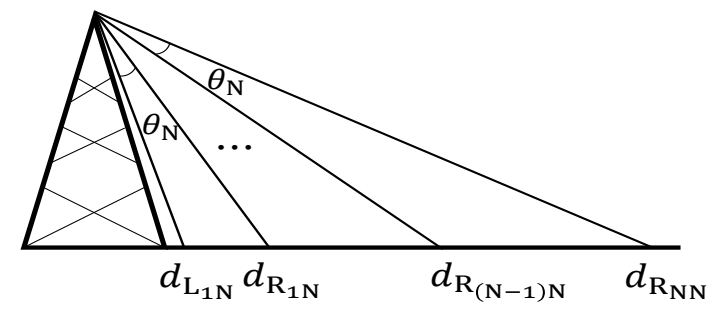

(b)

Figure 3. Illustration of the beam dictionary elements in case of (a) 2 beams

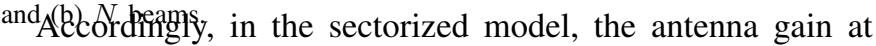
the $\mathrm{BS}$ side and user side $G_{x}\left(\theta_{x}\right)$, where $x \in\{\mathrm{B}, \mathrm{U}\}$, is given by [38]

$G_{x}\left(\theta_{x}\right)= \begin{cases}\gamma_{x}\left(\theta_{x}\right)=G_{0} \frac{2 \pi-\left(2 \pi-\theta_{x}\right) \epsilon}{\theta_{x}}, & \text { in the main lobe } \\ g=G_{0} \epsilon, & \text { otherwise }\end{cases}$

where $G_{0}$ is the antenna gain of an equivalent omnidirectional beam (i.e., $\theta_{x}=2 \pi$ ) and $\epsilon$ is a small positive constant $\ll 1$. In the ULA antenna model, each BS and user is assumed to be equipped with mm-wave ULA directional antennas consisting of $M_{B}$ and $M_{U}$ antenna elements respectively. Then, the BS antenna array response is:

$\boldsymbol{a}_{B}(\phi)=\frac{1}{\sqrt{M_{B}}}\left[1, e^{j \frac{2 \pi \kappa f_{c}}{c} \sin (\phi)}, \cdots, e^{j\left(M_{B}-1\right) \frac{2 \pi \kappa f_{c}}{c} \sin (\phi)}\right]$,

where $\kappa$ is the inter-element distance in the antenna system and $f_{c}$ is the center frequency of the mm-wave system. The user antenna response $\boldsymbol{a}_{U}(\psi)$ is simply obtained by replacing $\phi$ with $\psi$ and $M_{B}$ with $M_{U}$ in (4).

Let $\boldsymbol{w}\left(\theta_{B}\right) \in \mathbb{C}^{M_{B}}$ and $\boldsymbol{w}\left(\theta_{U}\right) \in \mathbb{C}^{M_{U}}$ represent the transmit and receive beamforming vectors. As defined in [14], the width of the beam can be controlled by changing the number of elements $M_{B}$ and $M_{U}$ in the antenna array. Then, the beamforming gains for the BS and the user are given by $G_{B}\left(\theta_{B}\right)=\left|\boldsymbol{a}_{B}^{H}(\phi) \boldsymbol{w}\left(\theta_{B}\right)\right|^{2}$ and $G_{U}\left(\theta_{U}\right)=\left|\boldsymbol{a}_{U}^{H}(\psi) \boldsymbol{w}\left(\theta_{U}\right)\right|^{2}$, respectively.

\section{Beam Dictionary}

We assume that each BS designs a sub- $6 \mathrm{GHz}$ assisted mmwave beamforming database. Specifically, each BS is capable of having beam dictionaries of different sizes, where each beam dictionary is composed by a set of beams characterized by the same width. The size of the beam dictionary denotes the number of beams that characterizes the dictionary. Furthermore, we assume that the main lobes of different beams of the same dictionary are non-overlapping. Together, the beams of a dictionary provide complete coverage in the geographical coverage area (i.e., the Voronoi cell) of the BS as shown in Fig. 3. Consequently, the larger the number of beams in the dictionary, the smaller is the beamwidth. It must be noted that for a typical BS deployed along the road, the neighbor BSs on either side may not be located at the same distances from it. As a result, the beam dictionary maintained at the BSs would contain the cell size information for both the sides of them. Without loss of generality, in what follows, we focus on one side of the typical BS.

Let $\theta_{1}=\arctan \left(\frac{d_{a}}{h_{B}}\right)-\arctan \left(\frac{d_{L_{11}}}{h_{B}}\right)$ be the beamwidth of the beam that provides total coverage of the area $d_{a}$, where $d_{L_{11}}=d_{L_{12}}=d_{L_{1 N}}=0$ is the starting point of the coverage area (as illustrated in Fig. 3) and $h_{B}$ is the height of the BS. Then, for the beam dictionary size $k$, the beamwidth is defined by $\theta_{k}=\theta_{1} / k$. Now, depending on this beamwidth $\theta_{k}$ and total number of beams, the left and right boundaries of each main lobe coverage positions of the $j$-th beam $(1 \leq j \leq k)$ are denoted as $d_{L_{j k}}$ and $d_{R_{j k}}$. The non-overlapping and adjacent assumption of the beams implies that $d_{R_{j k}}=d_{L_{(j+1) k}}, \forall j<k$.

Hence, we define the beam dictionary database $\mathcal{D B}$ of a mm-wave BS as a lower triangular matrix consisting of all feasible beams for each beam dictionary. Each element $\mathcal{D} \mathcal{B}_{k, j}$ of $\mathcal{D} \mathcal{B}$, where $j \leq k$, consists of a tuple $\left(\theta_{k}, d_{L_{j k}}, d_{R_{j k}}\right)$ corresponding to the $j$-th beam of the $k$-th beam dictionary. The elements of the tuple indicate respectively a) the width of the beam, b) the left boundary, and c) the right boundary of the main lobe of the beam (according to the sectorized model), as illustrated in (7). Then, for $k$-th beam dictionary, the $j$-th beam has a coverage area $\mathcal{C}_{j k}=d_{R_{j k}}-d_{L_{j k}}$. The steps for designing the beam dictionary at a mm-wave BS are:

1) After being deployed, the new BS exchanges inter-BS signals in the sub-6 $\mathrm{GHz}$ band to discover its geographical location on the street ${ }^{2}$, with respect to its neighbouring mm-wave $\mathrm{BSs}^{3}$. Using this information, a BS maps its own geographical coverage area with respect to its neighbors. As all the BSs are assumed to have the same transmit power, the cell boundaries are midway between two neighboring BSs as illustrated in Fig. 2.

2) For each value of beam dictionary $k \in\{1,2, \ldots, N\}$, the BS calculates the coverage areas of the associated beams as $\mathcal{C}_{j, k}=d_{R_{j k}}-d_{L_{j k}}$, where:

$d_{R_{j k}}=h_{B} \tan \left(\arctan \left(\frac{d_{L_{j k}}}{h_{B}}\right)+j \theta_{k}\right), \quad j=1, \cdots, k$,

$d_{L_{j k}}= \begin{cases}d_{R_{(j-1) k},} & j=2, \cdots, k, \\ 0, & j=1 .\end{cases}$

3) The resulting data-base is thus lower triangular matrix as follows:

$$
\mathcal{D B}=\left[\begin{array}{ccccc}
\left(\theta_{1}, d_{L_{11}}, d_{R_{11}}\right) & 0 & \ldots & 0 \\
\left(\theta_{2}, d_{L_{12}}, d_{R_{12}}\right) & 0 & \ldots & 0 & \\
\vdots & \vdots & \ddots & \ddots & \vdots \\
\left(\theta_{N}, d_{L_{1 N}}, d_{R 1 N}\right) & \ldots & \ldots & \left(\theta_{N}, d_{L_{N N}}, d_{R_{N N}}\right)
\end{array}\right],
$$

\footnotetext{
${ }^{2}$ Such prior geo-referencing, anyway required for mapping geographical coverage, can also be performed in alternative ways such as the GPS.

${ }^{3}$ This information can be provided a-priori by the operator during the deployment phase.
} 


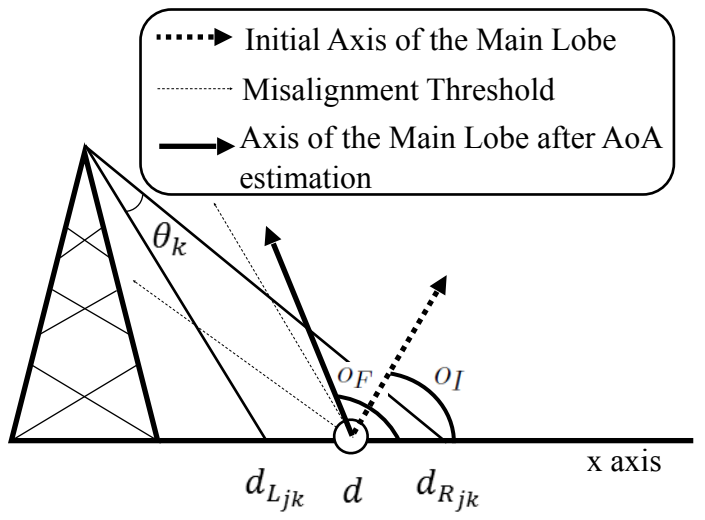

(b)

Figure 4. Illustration of the (a) beam selection error and (b) misalignment.

where the $k$-th row consists of the beam dictionary of size $k$ beams and contains the information about the width and the main lobe coverage areas of the corresponding beams.

Following the description of the beam-dictionary, we define two critical metrics of the system, which we will use to characterize the performance of the localization phase.

Definition 1. The beam-selection error is defined as the event that a UE located in $\mathcal{C}_{j, k}$ is estimated to be at $\hat{d}$, outside of $\mathcal{C}_{j, k}$, and accordingly, it is allotted a different beam than $\left(\theta_{j}, d_{L_{j, k}}, d_{R_{j, k}}\right)$. Let us denote as $\sigma_{d}^{2}$ the variance of the distance estimation error; the probability of beam-selection error $\left(\mathcal{P}_{B S}\right)$, given that the $U E$ is located at a distance $d$, is defined as

$$
\mathcal{P}_{B S, j, k}\left(d, \sigma_{d}^{2}\right)=\mathbb{P}\left(\hat{d}\left(d, \sigma_{d}^{2}\right) \notin \mathcal{C}_{j, k} \mid d \in \mathcal{C}_{j, k}\right) .
$$

This event is depicted in Fig. 4a.

Definition 2. The beam misalignment error is defined as the event that, after the AoA estimation, the UE beamforms towards a direction such that the axes of the main lobe of the UE and BS antennas have an angular separation greater than a predefined threshold $\nu$. Let us denote as o the initial user orientation and as $\hat{\psi}$ and $\sigma_{\psi}^{2}$ the estimated AoA and the variance of the AoA estimation error, respectively. After the AoA estimation, the user orients its main lobe towards the direction of $\hat{\psi}$ in order to align it towards the BS main lobe. The new orientation of the user main lobe is denoted by $o_{F}$ in Fig. 4b. The probability of misalignment error, given that the UE is located a distance d (i.e., $\left.\mathcal{P}_{M A}\right)$ is then defined as

$$
\mathcal{P}_{M A, j, k}\left(d, \psi, \sigma_{\psi}^{2}\right)=\mathbb{P}\left(\left|\psi-\hat{\psi}\left(d, \sigma_{\psi}^{2}\right)\right| \geq \nu\left(\theta_{k}, \theta_{U}\right)\right) .
$$

This event is depicted in Fig. 4b.

\section{Blockage, Path-Loss, and Signal Propagation}

Due to the presence of buildings and other obstacles, the communication links can either be in line of sight (LOS) or non line-of-sight (NLOS) state. We assume a LOS ball model for characterizing the blockage, similar to that in [10], with a LOS ball radius $d_{S}$. Thus, all the BSs present within a distance $d_{S}$ from the user are assumed to be in LOS, whereas, the BSs lying beyond $d_{S}$ are assumed to be in NLOS. Accordingly, the LOS BS process is denoted by $\xi_{L}$ and the NLOS BS process is denoted by $\xi_{N}$. Furthermore, because of the low local scattering in mm-wave communications, we consider a Nakagami fading $f$ with parameters $N_{L}$ and $N_{N}$ for the LOS and NLOS paths, respectively, and variance equal to 1 [39]. $N_{L}$ depends on the LOS paths, whereas, $N_{N}$ depends on the NLOS characteristics and specular reflection environment. Let us assume that $s(t)$ is the signal transmitted by the base station with power $P_{t}$. The power at the receiver located at a distance $d$ from the $\mathrm{BS}$ is hence given by $P_{r}=K P_{t}|f|^{2} G_{B}\left(\theta_{B}\right) G_{U}\left(\theta_{U}\right)\left(d^{2}+h_{B}^{2}\right)^{\frac{-\alpha}{2}}$, where $K$ is the path loss coefficient, and $\alpha$ is the path loss exponent. In our model, $\alpha=\alpha_{L}$ or $\alpha_{N}$ depending on whether the link is in LOS or NLOS state, respectively.

Let us assume that the received signal suffers from a zeromean additive Gaussian noise $n(t)$ with two-sided noise power spectral density of $N_{0}[\mathrm{dBm} / \mathrm{Hz}]$. Thus we can express the received signal as

$$
y(t)=\sqrt{P_{r}} s(t-\tau)+\sum_{i \in \mathcal{I}} \sqrt{P_{r, i}} s_{i}\left(t-\tau_{i}\right)+n(t),
$$

where $\mathcal{I}$ refers to the set of interfering BSs and accordingly $P_{r, i}=K P_{t}\left|f_{i}\right|^{2} g^{2}\left(h_{B}^{2}+d_{i}^{2}\right)^{-\frac{\alpha}{2}}, f_{i}, s_{i}(t)$ and $\tau_{i}$ represent the received signal power, channel coefficient, transmitted signal and delay corresponding to the $i$-th BS respectively. As a result the SINR in the data-communication phase is given by:

$$
\operatorname{SINR}_{C}=\frac{K P_{t}|f|^{2} G_{B}\left(\theta_{B}\right) G_{U}\left(\theta_{U}\right)\left(h_{B}^{2}+d^{2}\right)^{-\frac{\alpha}{2}}}{N_{0} B+\sum_{i \in \mathcal{I}} K P_{t}\left|f_{i}\right|^{2} g^{2}\left(h_{B}^{2}+d_{i}^{2}\right)^{-\frac{\alpha}{2}}} .
$$

In our analysis we have assumed that each BS divides its total transmit power equally among all the time frequency resources. Hence a user experiences interference from all the BSs in the network with the same transmit power. Power control and interference management strategies are interesting directions of research, which we will be treating in a future work.

Contrary to the data communication phase, in the localization phase, we do not consider the effect of interference. This is primarily because we assume that the localization estimation occurs using signals transmitted in the control channel, which is assumed to be interference-free due to the usage of orthogonal resources for transmitting the pilots. This is in line with classical and recent works on mm-wave localization [17], [40]. Additionally, unlike in the data service 


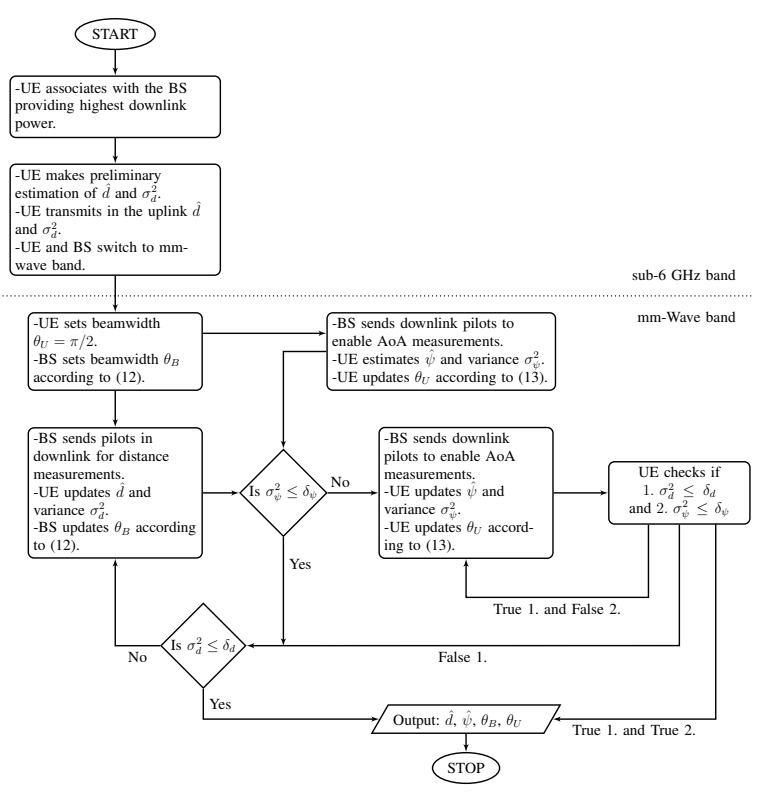

Figure 5. Flowchart representing the BS and user beam selection procedure.

phase, in order to simplify the CRLB derivation, we assume that the signal transmitted during the localization phase has a symmetric power spectral density [21] equal to $T_{s} / 2 \pi B$ where $T_{s}$ is the transmitted symbol duration.

Hence, the signal to noise ratio (SNR) in the localization process is given as:

$$
\mathrm{SNR}_{L}=\frac{K P_{t}|f|^{2} G_{B}\left(\theta_{B}\right) G_{U}\left(\theta_{U}\right)}{N_{0} B}\left(h_{B}^{2}+d^{2}\right)^{-\frac{\alpha}{2}} .
$$

\section{Initial Beam-Selection Procedure}

In this section, we discuss our initial beam-selection procedure for a user arriving in the mm-wave network. In this procedure, the BS and the user select appropriate beam pairs, $\theta_{B}$ and $\theta_{U}$ respectively, based on the localization accuracy required for the initial access.

1) When a new user arrives in the network, it associates with the BS that provides the highest downlink received power in the sub-6GHz band. The UE then makes a coarse initial estimation $\hat{d}$ of its position which is characterized by an estimation-error variance $\sigma_{d}^{2}$. Without loss of generality, this initial localization can be obtained by means of technologies such as sub-6 GHz band (e.g., based on RSSI or ToF measurements), external means such as GPS or WiFi or even with standalone mm-wave band based distance estimation. The UE then relays this information to the BS.

2) Next, the BS and the UE switch to the mm-wave band. The UE selects a mm-wave beam of beamwidth $\theta_{U}$, initially quasi-omnidirectional (with beamwidth $\pi / 2$ ).

3) In $\mathcal{D B}$, for each beam dictionary $k$, there exists a beam $j$ such that $d_{L_{j k}} \leq \hat{d} \leq d_{R_{j k}}$. Out of all such possible beam and beamwidth pairs $j$ and $k$, the BS selects the pair with the largest beam dictionary size (i.e., the thinnest beam) that results in a beam-selection error probability
$\mathcal{P}_{B S, j, k}\left(d, \sigma_{d}^{2}\right)$ less than a threshold $\delta_{B S}$. Mathematically, $\theta_{B}=\theta_{k}$ such that

$$
\begin{array}{r}
k=\max (i): \mathcal{P}_{B S, j, i}\left(d, \sigma_{d}^{2}\right) \leq \delta_{B S}, i=1,2, \ldots, N, \\
d_{L_{j k}} \leq \hat{d} \leq d_{R_{j k}} .
\end{array}
$$

The expression for beam-selection error is derived in Lemma 2.

4) After this step, the BS sends downlink pilots in mm-wave band, the UE updates $\hat{d}$ and $\sigma_{d}^{2}$ and transmits this information in the uplink. The BS then updates $\theta_{B}$ accordingly.

5) In parallel with the ranging estimation, the UE also measures the AoA of the BS signal $\hat{\psi}$, which is characterized by an estimation-error variance $\sigma_{\psi}^{2}$. First, the user sets the angle of the maximum gain equal to $\hat{\psi}$; then, it fixes $\theta_{U}$ as the thinnest beam $\theta_{i}$ for which the misalignment error probability $\mathcal{P}_{M A, j, k}\left(d, \psi, \sigma_{\psi}^{2}\right)$ is less than a threshold $\delta_{M A}$, given that the BS selects the $j$-th beam of size $\theta_{k}$. Mathematically,

$$
\begin{array}{r}
\theta_{U}=\min \left(\theta_{i}\right):\left[\mathcal{P}_{M A, j, k}\left(d, \psi, \sigma_{\psi}^{2}\right) \leq \delta_{M A}\right], \text { and } \\
0 \leq \theta_{i} \leq \frac{\pi}{2} .
\end{array}
$$

The expression for misalignment error is derived in Lemma 3.

6) Let $\delta_{\psi}$ and $\delta_{d}$ be the localization accuracy requirements for reliable initial access; the refinement procedure terminates when either i) the BS beam and the UE beam simultaneously satisfy $\sigma_{d}^{2} \leq \delta_{d}$ and $\sigma_{\psi}^{2} \leq \delta_{\psi}$ or ii) a maximum number of iterations is reached.

7) When the termination conditions are not satisfied, the UE continues to measure the downlink pilots, and accordingly, the estimates of $\hat{d}, \sigma_{d}^{2}, \hat{\psi}$, and $\sigma_{\psi}^{2}$ are updated. Following these new estimates, steps 3 and 5 are repeated for an improved initial beam selection.

We refer the reader to Fig. 5 for a description of the steps involved in the iterative loop for the initial access. It must be noted that the number of steps the initial beam-selection algorithm takes to terminate depends directly on the desired resolution of the localization. In other words, the more stringent the localization requirements of the initial access are, the more will be the number of steps of the initial beamselection algorithm. Consequently, by tuning $\delta_{d}$ and $\delta_{\psi}$, the initial access delay can be controlled. There is thus an inherent trade-off between initial access delay and the accuracy of the UE localization, which we shall discuss in Section V.

The proposed initial-access scheme improves the latency for establishing mm-wave connection in the system as compared to an exhaustive search solution (as we will see in the numerical results). However, in our proposed method, even though we minimize the probability of beam selection and misalignment errors in every iteration, there is still a small chance that the beams are not aligned. In this case, a resynchronization procedure is required. In addition, in case the direct path between the user and the BS gets obstructed due to dynamic blockage, the localization performance would suffer and the system could experience beam-selection errors. With 
our algorithm, this situation can be prevented by adapting the beam size using the previously stored location estimate and the current estimation accuracy. Thus, integrating the estimation accuracy (e.g., the variance of the estimation error) enables a fall-back solution that is not possible when using only location estimate. In case of using a simple exhaustive search, the entire set of beam combinations from the UE and the BS sides needs to be checked to re-estabish the connection.

\section{Performance Characterization of the LOCALIZATION AND DATA PHASES}

After the initial access phase, the system starts the service phase, which comprises two alternating phases: the data phase and the localization phase (see Fig. 1). In the localization phase, mm-wave transmission is used to update the estimates of distance $\hat{d}$ and AoA of the signal received at the user $\hat{\psi}$, and potentially improve the localization accuracy. In the data phase, the UE is served by the BS with a mm-wave beam, which is selected from the dictionary according to the estimated user location. We propose a framework where the radio frames are divided into flexible sub-frames in order to address jointly the requirements of localization and data services. In this section, we mathematically characterize the performance metrics of localization (v.i.z., position and orientation accuracy) and communication (in terms of downlink rate coverage), as a function of the resource partitioning factor $(\beta)$ and the sizes of the beams $\left(\theta_{U}, \theta_{B}\right)$, in order to optimize the radio frame design.

\section{A. Localization Phase}

We model the accuracy of the localization phase in terms of the variances of the estimated distance of the UE from the BS $\hat{d}$ and of the AoA $\hat{\psi}$ characterized by the CRLB ${ }^{4}$. CRLB provides us with a lower bound on the variance of unbiased estimators for those two variables. It is defined as the inverse of Fisher Information Matrix (FIM), which measures the amount of information on each of the estimation variables present in the observed signal, given a priori statistics for the latter. Then, using these tools, we characterize the beam-selection error $\mathcal{P}_{B S}$ resulting from a distance estimation error and we model the misalignment $\mathcal{P}_{M A}$ between the user and the $\mathrm{BS}$ due to inaccuracy in the estimation of the AoA.

1) CRLB of the Estimation Parameters: Let the estimates be represented by the vector $\boldsymbol{\eta}=\left[d, \psi, f_{R}, f_{I}\right]$, where $f_{R}$ and $f_{I}$ respectively describe the real and imaginary parts of the channel between the UE and the serving BS.

Lemma 1. The CRLBs for the estimation of the distance and the AoA can be written as follows:

$$
\begin{aligned}
& \sigma_{d}^{2}=\left(\zeta G_{U}\left(\theta_{U}\right) G_{B}\left(\theta_{B}\right) \frac{B^{2} \pi^{2}}{3 c^{2}}\right)^{-1}, \\
& \sigma_{\psi}^{2}=\left(\zeta G _ { B } ( \theta _ { B } ) \left(\left|\dot{\boldsymbol{a}}_{U}^{H}(\psi) \boldsymbol{w}_{U}\left(\theta_{U}\right)\right|^{2}-\right.\right. \\
& \left.\left.\frac{\left|\boldsymbol{a}_{U}^{H}(\psi) \boldsymbol{w}_{U}\left(\theta_{U}\right) \boldsymbol{w}_{U}^{H}\left(\theta_{U}\right) \dot{\boldsymbol{a}}_{U}(\psi)\right|^{2}}{G_{U}\left(\theta_{U}\right)}\right)\right)^{-1},
\end{aligned}
$$

${ }^{4}$ For the purpose of analysis, in this paper, we consider the bound to represent the variance. However, in practice, we can use the actual variance of estimation depending on the estimator used. where $\zeta=\frac{2 S N R_{L} B(1-\beta) T_{F}}{G_{B}\left(\theta_{B}\right) G_{U}\left(\theta_{U}\right)}, c$ is the speed of light, $B$ is the bandwidth and $\dot{\boldsymbol{a}}_{U}(\psi)=\partial \boldsymbol{a}_{U}(\psi) / \partial \psi$. Also, $\mathbb{R}\{$.$\} and \mathbb{I}\{$. represent the real and imaginary operators.

Proof. See [17], [40].

Remark 1. Naturally, the localization accuracy depends on the temporal resources allotted to the localization phase, i.e., $(1-\beta) T_{F}$. In particular, we note from Lemma 1 that the CRLBs of the estimation of the distance and the AoA are inversely proportional to $\zeta$. Thus, the variance of the error in estimation decreases with increasing $S N R_{L}$ and decreasing $\beta$. Accordingly, the higher the transmit power and/or the BS deployment density, the better the estimation performance. Similarly, larger bandwidth improves the distance estimation as it provides finer resolution for accurately analyzing the time of arrival of the received signal.

2) Beam-Selection Error: Without loss of generality, assume that the real position of the UE is $d_{L_{j k}} \leq d \leq d_{R_{j k}}$, and accordingly, for a given beam dictionary $k$, the $j$-th beam, whose coverage area is given by $\mathcal{C}_{j, k}=d_{R_{j k}}-d_{L_{j k}}$, should be assigned to it. However, due to ranging errors, the estimated position of the user $\hat{d}$ is distributed as $\mathcal{N}\left(d, \sigma_{d}^{2}\right)$, where $\sigma_{d}^{2}$ is defined in (15a). Hence, a beam-selection error occurs for the user when $\hat{d}$ is not inside the correct interval defined by $d_{L_{j k}}$ and $d_{R_{j k}}$ (see Fig. 4a). Averaging out on the possible beams that can be selected depending on the relative positions of the typical user to $\mathrm{BS}$, we have the following result.

Lemma 2. The probability of beam-selection error when the $B S$ estimates the UE to be in the position $\hat{d}$ and selects a beam of width $\theta_{k}$, is computed as:

$$
\begin{aligned}
\mathcal{P}_{B S, j, k}\left(d, \sigma_{d}^{2}\right) & =\mathbb{P}\left(\hat{d}<d_{L_{j k}}\right)+\mathbb{P}\left(\hat{d}>d_{R_{j k}}\right) \\
& =1-\mathcal{Q}\left(\frac{d_{L_{j k}}-d}{\sigma_{d}}\right)+\mathcal{Q}\left(\frac{d_{R_{j k}}-d}{\sigma_{d}}\right),
\end{aligned}
$$

where $\mathcal{Q}(\cdot)$ is the $Q$-function. Accordingly, the average beamselection error over all the possible UE positions in case of a total number of beams $N(y)$ (where $y$ is the cell-size) with beamwidth $\theta_{k}$ is given by:

$\overline{\mathcal{P}}_{B S}=\int_{0}^{\infty}\left(\sum_{j=1}^{N(y)} \int_{d_{L_{j k}}}^{d_{R_{j k}}} \mathcal{P}_{B S, j, k}\left(x, \sigma_{d}^{2}\right) f_{d}(x) d x\right) f_{d_{a}}(y) d y$.

Proof. See Appendix A.

Corollary 1. In case of deterministic deployments, where the BSs are equispaced, (17) becomes:

$$
\begin{aligned}
& \overline{\mathcal{P}}_{B S}=\sum_{j=1}^{N} \int_{d_{L_{j N}}}^{d_{R_{j N}}} \mathcal{P}_{B S, j, N}\left(x, \sigma_{d}^{2}\right) f_{d}(x) d x, \quad \text { where, } \\
& N=\left\lceil\frac{1}{\theta_{N}} \arctan \left(\frac{\frac{1}{\lambda}-d_{L_{1 N}}}{h_{B}}\right)\right] .
\end{aligned}
$$


Here we note that for the initial deployments of the mm-wave BSs [34], this special case if of particular importance.

Remark 2. The wider the antenna beam, the larger is the value of $\mathcal{C}_{j, k}$. Thus, for a given distance estimation accuracy (i.e., $\left.\sigma_{d}\right)$, the beam selection error is smaller for a larger beamwidth since $\mathcal{P}_{B S, j, k}\left(x, \sigma_{d}^{2}\right)$ decreases with $\mathcal{C}_{j, k}$ in (16). On the other hand, with increasing $\theta_{k}$, the value of $\sigma_{d}$ increases because of the lower antenna gain. This increases the beam selection error. Overall, this results in the peaky behaviour of the beam selection error that we observe in the results.

3) Misalignment Error: We assume that the UE estimates the AoA and then sets the axis of the main lobe of its antenna to $\hat{\psi}$. However, in case of erroneous estimate, there exists a possibility of error in alignment of the beams (see Fig. 4b). Let us assume that the user located at a distance $d$ from the $\mathrm{BS}$ has an AoA $\psi$ with respect to the $\mathrm{BS}$, and that is served by the $j$-th beam of size $\theta_{k}$ (i.e., $\theta_{B}=\theta_{k}$ ). Due to the noise affecting the received signal, the estimated AoA $\hat{\psi}$ is affected by random errors. Consequently, we assume that $\hat{\psi}$ is distributed as $\mathcal{N}\left(\psi, \sigma_{\psi}^{2}\right)$, where $\sigma_{\psi}^{2}$ is defined in (15b). For our analysis, we define the BS-UE beam pair to be misaligned, if $|\psi-\hat{\psi}|$ is larger than a threshold $\nu\left(\theta_{B}, \theta_{U}\right)$. In other words, in case the axes of the main lobe of the beams of the UE and the BS have an angular separation larger than the a-priori angular threshold $\nu\left(\theta_{B}, \theta_{U}\right)$, we assume that the beams are misaligned.

Lemma 3. The misalignment error probability for a UE at a distance $x$ from the $B S$ is given by

$$
\begin{aligned}
\mathcal{P}_{M A, j, k}\left(d, \psi, \sigma_{\psi}^{2}\right) & =\mathbb{P}(\psi-\hat{\psi} \leq-\nu)+\mathbb{P}(\psi-\hat{\psi} \geq \nu) \\
& =2 \mathcal{Q}\left(\frac{\nu}{\sigma_{\psi}}\right) .
\end{aligned}
$$

Then, the average misalignment probability is calculated by taking the expectation with respect to $d$ and $\psi$, i.e., $\overline{\mathcal{P}}_{M A}=$ $\mathbb{E}_{d, \psi}\left[\mathcal{P}_{M A}\left(d, \psi, \sigma_{\psi}^{2}\right)\right]$, where the distribution of $d$ is $f_{d}(y)$ (see Section II-A), and the distribution of $\psi$ is uniform between 0 and $2 \pi$.

Proof. The proof follows similar to Lemma 2.

Remark 3. From (18), it can be observed that the larger the threshold for misalignment, the lower is the misalignment probability. As the threshold is directly related to the transmit and receive beamwidths, in case of wider beamwidths, the probability of misalignment is lower.

Remark 4. It can be noted that the expressions for the beam selection and misalignment errors can be evaluated into a closed form for the deterministic case with the assumption that the variance of the range and orientation is independent of the distance. Accordingly, the expression for the coverage probability reduces to a single integral. Furthermore, based on the deployment scenario, appropriate approximation of the Gaussian Q-function (e.g., [41], [42]) can be employed to simplify the integrals. However, we do not discuss these special cases so as to focus on the main objective of the paper, i.e., the interplay of the communication and localization performance.

\section{B. Data Phase}

In this section, first we characterize the performance of the typical UE considering beam-selection and misalignment errors. Then, we propose a methodology to jointly configure the split between the localization and data phases as well as the BS beam in order to optimize data and localization performance simultaneously. Accordingly, in the following, we first model the effective SINR coverage probability and then we define the effective user data-rate.

1) Effective SINR Coverage Probability: Since the locations of the BSs are modeled as points of a 1D PPP, the locations of the users are assumed to be uniformly in the coverage area of the BSs, and the orientation of the users is assumed to be uniformly distributed between 0 and $2 \pi$, the SINR of a user is a random variable. The SINR coverage probability is defined as the probability that the typical UE receives an SINR over a given threshold $T$. From the network perspective, it represents the fraction of total users under coverage. The SINR coverage probability is defined as the probability that the typical UE receives an SINR over a given threshold $T$. From the network perspective, it represents the fraction of total users under coverage. Mathematically, it is characterized in the following theorem.

Theorem 1. The SINR coverage probability of the typical user $\mathcal{P}_{C}\left(T, j, \theta_{k}, \theta_{U}\right)$ served by the $j$-th beam of width $\theta_{k}$ is given by:

$$
\begin{aligned}
& \mathcal{P}_{C}\left(T, j, \theta_{k}, \theta_{U}\right)=\int_{0}^{2 \pi} \int_{d_{L_{j, k}}}^{d_{R_{j, k}}}\left[\mathcal{P}_{B S, j, k}\left(x, \sigma_{d}^{2}\right) \mathcal{T}_{B S}(x, T)+\right. \\
& \left(1-\mathcal{P}_{B S, j, k}\left(x, \sigma_{d}^{2}\right)\right)\left(\left(1-\mathcal{P}_{M A, j, k}\left(x, \psi, \sigma_{\psi}^{2}\right)\right) \mathcal{T}_{0}(x, T)+\right. \\
& \left.\left.\mathcal{P}_{M A, j, k}\left(x, \psi, \sigma_{\psi}^{2}\right) \mathcal{T}_{M A}(x, T)\right)\right] f_{d}(x) f(\psi) d x d \psi
\end{aligned}
$$

where

$$
\begin{aligned}
& \mathcal{T}_{0}(x, T)=\sum_{n=1}^{N_{L}}(-1)^{n+1}\left(\begin{array}{c}
N_{L} \\
n
\end{array}\right) \\
& \exp \left(-\left(\frac{n \eta_{L} T N_{0}}{P_{t} K \gamma_{B}\left(\theta_{k}\right) \gamma_{U}\left(\theta_{U}\right) z^{-\alpha_{L}}}+\mathcal{A}_{L 0}(x, T)+\mathcal{A}_{N 0}(x, T)\right)\right) \\
& \mathcal{T}_{M A}(x, T)=\sum_{n=1}^{N_{L}}(-1)^{n+1}\left(\begin{array}{c}
N_{L} \\
n
\end{array}\right) \\
& \exp \left(-\left(\frac{n \eta_{L} T N_{0}}{P_{t} K \gamma_{B}\left(\theta_{k}\right) g z^{-\alpha_{L}}}+\mathcal{A}_{L M A}(x, T)+\mathcal{A}_{N M A}(x, T)\right)\right) \\
& \mathcal{T}_{B S}(x, T)=\sum_{n=1}^{N_{L}}(-1)^{n+1}\left(\begin{array}{c}
N_{L} \\
n
\end{array}\right) \\
& \exp \left(-\left(\frac{n \eta_{L} T N_{0}}{P_{t} K g^{2} z^{-\alpha}}+\mathcal{A}_{L B S}(x, T)+\mathcal{A}_{N B S}(x, T)\right)\right)
\end{aligned}
$$

in which $z=\sqrt{x^{2}+h_{B}^{2}}, \sigma_{d}^{2}$ is a function of $x, \sigma_{\psi}^{2}$ is a function of $x$ and $\psi$, and

$$
\mathcal{A}_{L 0}(x, T)=2 \lambda \int_{x}^{d_{S}} 1-\frac{1}{\left(1+\frac{\eta_{L} T g^{2} q^{-\alpha_{L} 2 \lambda y}}{N_{L} \gamma_{B}\left(\theta_{k}\right) \gamma_{U}\left(\theta_{U}\right) z^{-\alpha_{L}}}\right)^{N_{L}}} d y,
$$




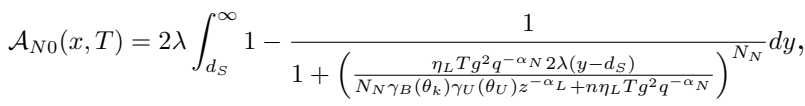

$$
\begin{aligned}
& \mathcal{A}_{L M A}(x, T)=2 \lambda \int_{x}^{d_{S}} 1-\frac{1}{\left(1+\frac{\eta_{L} T g q^{-\alpha_{L} 2 \lambda y}}{N_{L} \gamma_{B}\left(\theta_{k}\right) z^{-\alpha_{L}}}\right)^{N_{L}}} d y,
\end{aligned}
$$

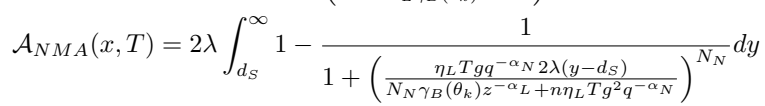

$$
\begin{aligned}
& \mathcal{A}_{L B S}(x, T)=2 \lambda \int_{x}^{d_{S}} 1-\frac{1}{\left(1+\frac{\eta_{L} T q^{-\alpha_{L} 2 \lambda y}}{N_{L} z^{-\alpha_{L}}}\right)^{N_{L}}} d y,
\end{aligned}
$$

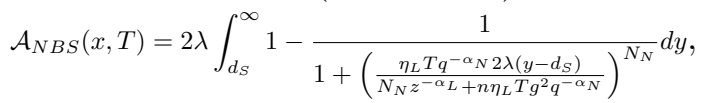

and $q=\sqrt{y^{2}+h_{B}^{2}}$.

Proof. See Appendix B.

In (19), the term $\mathcal{T}_{0}(x, T)$ corresponds to the case in which there is no beam-selection error as well as no misalignment. In this case, we have $G_{B}\left(\theta_{k}\right)=\gamma_{B}\left(\theta_{k}\right)$ and $G_{U}\left(\theta_{U}\right)=\gamma_{U}\left(\theta_{U}\right)$ resulting in a high coverage probability. The term $\mathcal{T}_{M A}(x, T)$ represents the case where there is no beam-selection error, but the BS-user beam pair suffers from misalignment. Here the coverage probability decreases as compared to $\mathcal{T}_{0}(x, T)$ although $G_{B}\left(\theta_{B}\right)$ remains the same, since here we have $G_{U}\left(\theta_{U}\right)=g$. Finally, the term $\mathcal{T}_{B S}(x, T)$ refers to the case when there is a beam-selection error. It must be noted that according to our assumption, in the case of beam-selection error, we assume that the beams are always misaligned. Here we have $G_{B}\left(\theta_{k}\right)=G_{U}\left(\theta_{U}\right)=g$. In case of exhaustivesearch, the users will not suffer from beam-selection or misalignment errors, i.e., for exhaustive-search, in (19) we have $\mathcal{P}_{B S, j, k}\left(x, \sigma_{x}^{2}\right)=0$ and $\mathcal{P}_{M A, j, k}\left(x, \psi, \sigma_{\psi}^{2}\right)=0$. Accordingly, the users will experience a better SINR, as discussed in the following proposition:

Proposition 1. For a given value of $\theta_{k}$ and $\theta_{U}$, an exhaustivesearch based initial access algorithm will suffer from no beamselection error and no misalignment error. Consequently, the SINR coverage probability for an exhaustive search algorithm is given by:

$$
\mathcal{P}_{C}\left(T, j, \theta_{k}, \theta_{U}\right)=\int_{0}^{2 \pi} \int_{d_{L_{j, k}}}^{d_{R_{j, k}}} \mathcal{T}_{0}(x, T) f_{d}(x) f(\psi) d x d \psi
$$

Corollary 2. The overall SINR coverage probability, considering all the $N$ beams of size $\theta_{k}$ is:

$$
\overline{\mathcal{P}}_{C}\left(T, \theta_{k}, \theta_{U}\right)=\mathbb{E}_{d_{a}}\left[\sum_{j=1}^{N\left(d_{a}\right)} \mathcal{P}_{C}\left(T, j, \theta_{k}, \theta_{U}\right)\right],
$$

where the expectation is taken with respect to the inter-BS distance $d_{a}$ given by (1).

2) Effective Rate Coverage Probability: Let $B$ denote the system bandwidth and $T_{I}$ the duration of the initial access procedure. As the data phase uses $\beta$ fraction of the total resources in the service phase $T_{F}$, we can compute the probability $\mathcal{P}_{R}\left(r_{0}, \beta, \theta_{k}, \theta_{U}\right)$ that the effective rate is above given threshold $r_{0}$ as below.
Lemma 4. For a given SINR coverage probability, the effective rate coverage probability is given by $\overline{\mathcal{P}}_{C}\left(2^{\frac{r_{0}\left(T_{T}+T_{F}\right)}{\beta T_{F} B}}-1, \theta_{k}, \theta_{U}\right)$, where $r_{0}$ is the target rate threshold.

Proof.

$$
\begin{aligned}
\overline{\mathcal{P}}_{R}\left(r_{0}, \beta, \theta_{k}, \theta_{U}\right) & =\mathbb{P}\left(\frac{\beta T_{F}}{T_{I}+T_{F}} B \log _{2}\left(1+S I N R_{C}\right) \geq r_{0}\right) \\
& =\mathbb{P}\left(S I N R_{C} \geq 2^{\frac{r_{0}\left(T_{I}+T_{F}\right)}{\beta T_{F} B}}-1\right) \\
& =\overline{\mathcal{P}}_{C}\left(2^{\frac{r_{0}\left(T_{I}+T_{F}\right)}{\beta T_{F} B}}-1, \theta_{k}, \theta_{U}\right)
\end{aligned}
$$

\section{Joint Optimization of the Transmit Beamwidth and Radio}

Frame Structure

Given the characterization of the effective rate coverage probability, we present a schematic for selecting, at the BS, the optimal beam from the designed beam dictionary. The proposed schematic is presented in the form of a two-stage optimization problem as:

$$
\theta^{*} \underset{\theta_{k}}{\operatorname{argmax}}\left[\begin{array}{cc}
\max _{\beta} & \overline{\mathcal{P}}_{R}\left(r_{0}, \beta, \theta_{k}, \theta_{U}\right) \\
\text { subject to } & \overline{\mathcal{P}}_{B S} \leq \epsilon \\
& \overline{\mathcal{P}}_{M A} \leq \epsilon^{\prime}
\end{array}\right] .
$$

We obtain the optimal $(\theta, \beta)$ pair using an exhaustive search on a finite set of possible beamwidth and resource partitioning factors. In particular, in the first step, for a given $\theta_{k}$, we select the value of $\beta_{k}^{*}$ that maximizes the effective rate coverage probability subject to apriori constraints $\epsilon$ and $\epsilon^{\prime}$, on the beam-selection and the misalignment errors, respectively. These constraints are system parameters which are governed by the accuracy requirements for the localization service. In the subsequent frames, based on the new measurements, the estimates $(\hat{d}$ and $\hat{\psi})$ are updated and the measurement error variances $\left(\sigma_{d}^{2}\right.$ and $\sigma_{\psi}^{2}$ ) change. Accordingly, the BS beamwidth $\theta^{*}$ can be further updated by using (23).

We emphasize that the optimal beamwidth thus calculated is different from the adaptive beamwidth value evaluated in the initial access phase. The former is calculated offline to maximize the data-rate given a set of system parameters, whereas, the latter is the beamwidth adapted to achieve the required resolution in terms of distance and orientation.

\section{Numerical Results AND Discussion}

Now we present numerical results related to the initial beam-selection and the localization-communication trade-offs developed in this paper. The parameter values are given in Table 1 . The numerical results follow the analytical expressions derived in this paper, where the beam-selection and misalignment errors are characterized by (16) and (18), respectively. The errors are incorporated into the SINR coverage probability expressions as derived in Theorem 1. Leveraging this, the rate coverage probability follows (21).

\section{A. Performance of the Initial Access Phase}

First, let us discuss the performance of the initial beamselection strategy developed in Section III. In Fig. 6a, we plot the enhancement in positioning resolution (characterized as

\section{ACCEPTED MANUSCRIPT}




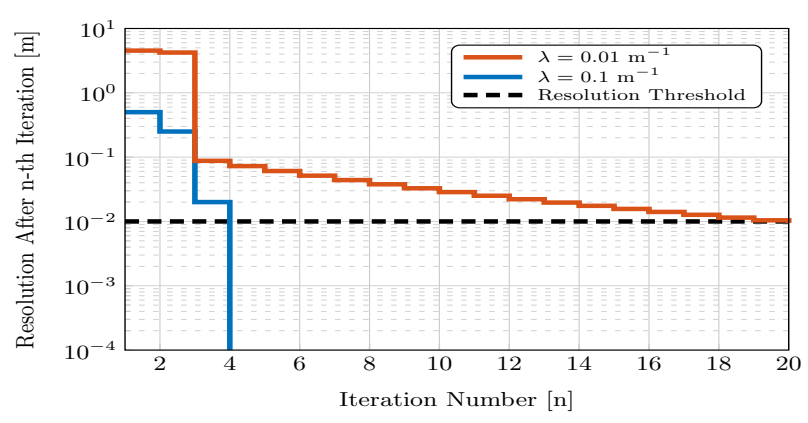

(a)

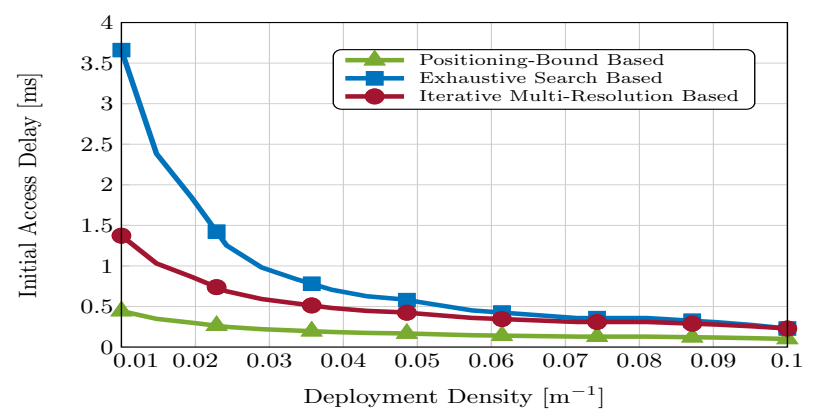

(b)

Figure 6. (a) Resolution in the $n$-th step of the localization-based initial access strategy for different deployment densities, and (b) Comparison of the delay in initial access of our localization-based strategy to the iterative and exhaustive search strategies.

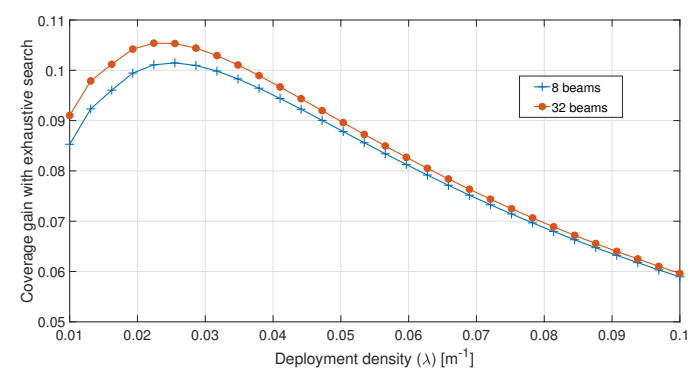

Figure 7. Gain in SINR coverage with an exhaustive search based initialaccess algorithm for two beam dictionary sizes.

the variance of the ranging error) with increasing the number of steps of our initial beam-selection algorithm. Here, we have assumed that $\delta_{d}=0.01 \mathrm{~m}$ is the minimum resolution required to provide mm-wave date-service. As expected (see Remark 1), we note that for denser small cell deployments (e.g., $\lambda=0.1 \mathrm{~m}^{-1}$ ) the algorithm stops at a lower number (here 4) of iterations, as compared to the sparser deployment scenarios. As the deployment becomes sparser (e.g., $\lambda=0.01$ $\mathrm{m}^{-1}$ ), a larger number of steps is required for the initial access procedure. This is precisely due to the fact that for denser deployments, $\mathrm{SNR}_{L}$ increases. Accordingly, a larger beamwidth is sufficient and hence, a lower number of iterations are required to meet the localization requirements.

Then, in Fig. 6b we compare the initial access delay of the proposed localization-bound based strategy with the one achieved by two well-known beam-sweeping solutions: exhaustive search and iterative search [13], [43]. For the exhaustive search, we consider the beamwidth of the BS and the UE to be fixed and equal to $\theta_{B}$ and $\theta_{U}$, respectively. Thus, the BS and the UE go through all the possible $\frac{2 \pi}{\theta_{B}} \times \frac{2 \pi}{\theta_{U}}$ beam combinations to select the beam pair that maximizes the SNR. The exhaustive search has been adopted in the standards IEEE 802.15.3c and IEEE 802.11ad [44], [45]. On the other hand, for the case of iterative search (similar to bisection search in [46]), we assume that the BS initiates the procedure with $k=2$ while the user uses an omni-directional beam. Out of the two possible beams, the BS identifies the beam that results in the highest downlink SNR and changes its search space to the region covered by that beam. Then, the BS changes its beam size to a thinner one (of dictionary $k=4$ ) and uses 2 out of the 4 beams from the dictionary which lie within the modified search space. We assume that the initial beam-selection phase terminates when this process chooses the same beamwidth $\theta_{B}$ selected by our algorithm. Thereafter, the BS fixes the selected beam and the UE carries out the same procedure for obtaining the user side beam. In our system, similar to [13], we assume that i) one OFDM symbol length (including cyclic prefix) is $14.3 \mu \mathrm{s}$, ii) each synchronization signal occupies only one OFDM symbol, and iii) the beam reference signal is also transmitted in the same symbol to uniquely identify the beam index. Clearly, our strategy provides considerably faster initial access precisely due to the smaller number of steps than those required by the exhaustive and iterative search based schemes.

The number of iterations our algorithm takes to terminate is a direct measure of the delay in the initial beam-selection procedure. Specifically, we assume that this delay is computed as the product of the sum of the required number of steps at the BS side and the UE side and the duration of one OFDM symbol. In Fig. 6a, with $\lambda=0.01 \mathrm{~m}^{-1}$, we observe that for a required $\delta_{d}=0.01 \mathrm{~m}$, our algorithm terminates in 20 steps, which corresponds to a delay of about 5.7 ms. Wheras, if the positioning requirement was specified to be $0.1 \mathrm{~m}$, the algorithm would have terminated in 3 steps, which corresponds to a initial beam-selection delay of about $1 \mathrm{~ms}$. Thus, there exists a fundamental trade-off between the localization requirement $\left(\delta_{d}\right.$ and $\left.\delta_{\psi}\right)$ and the delay in the initial beam-selection.

For a fair comparison, we emphasize that classical algorithms such as the exhaustive search do not suffer from beam-selection and misalignment errors. This is shown in Fig. 7, where we plot the gain in SINR coverage with an exhaustive search based initial-access algorithm as compared to our proposed algorithm for two beam dictionary sizes. We observe that with a large number of beams, the SINR gain increases. This is precisely because a large beam dictionary size leads to smaller beam coverage, which in turn increases the beam selection error. More interestingly, we see that for dense deployment of BSs, the gain drops dramatically as the beam-selection and misalignment errors with the proposed initial-access scheme are limited.

\section{B. Performance of the Localization Phase}

The reduction in the initial beam-selection delay with the proposed algorithm is naturally associated with localization errors, which we discuss in this section.

In Fig. $8 \mathrm{a}$ we plot the beam selection error as a function of the beam-dictionary size $(N)$ for different antenna gains. It must be noted that the effect of a larger beamwidth on the beam-selection error is non-trivial (see Remark 2). Larger

\section{ACCEPTED MANUSCRIPT}




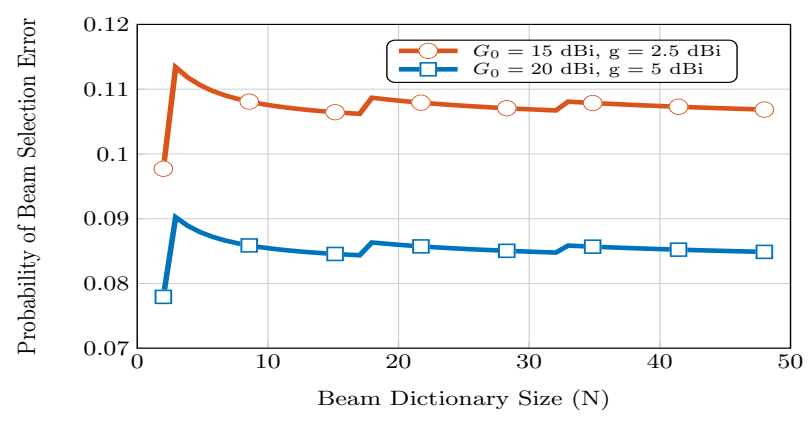

(a)

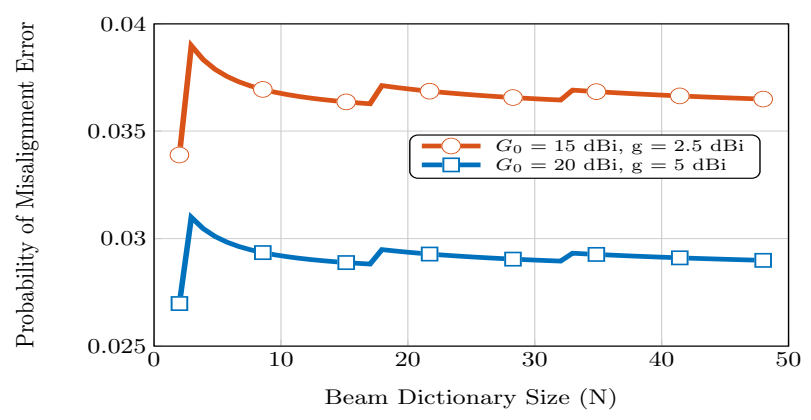

(b)

Figure 8. (a) Probability of beam selection error vs the beam dictionary size for different antenna gains; (b) Probability of misalignment error vs the beam dictionary size for different antenna gains.

beamwidth results in a lower radiated power, which leads to a higher CRLB for distance estimation, which may lead to a higher beam-selection error. However, a larger beamwidth also corresponds to a larger geographical area covered on ground by the beam (i.e., larger $\mathcal{C}_{k, j}$ ), which leads to a lower beamselection error.

As expected, the beam-selection error is minimized for $N=1$, when a single beam encompasses all the cell coverage area of the BS. The beam-selection error would occur only when using a distance-based cell selection scheme, if the actual position of the UE is outside the coverage area of the serving BS. For $N \geq 2$, interestingly, we observe a stepped behavior of the probability of beam selection error with respect to the beam dictionary size. The beam selection error gradually decreases with increasing beam dictionary size due to the increasing antenna gain (see eq. (3)). This behaviour continues until a certain value of beam dictionary size, where the beam width becomes so thin that the probability that the user lies outside the beam coverage area is high. This results in an increase in the probability of beam-selection error, which then gradually decreases, when increasing the beam dictionary size, and so on. This brings forth an important characteristic of the system: for achieving a given beam selection error performance, multiple beam sizes can exist. This is precisely because of the fact that with the decreasing size of the beams, two conflicting phenomena occur: i) an improvement in the estimation performance owing to larger antenna gain and ii) a reduction of the geographical area covered by each beam.

Fig. $8 \mathrm{~b}$ shows that the beam misalignment probability has the same peaky trend of the beam selection error with respect to the beam dictionary size. Specifically, the misalignment

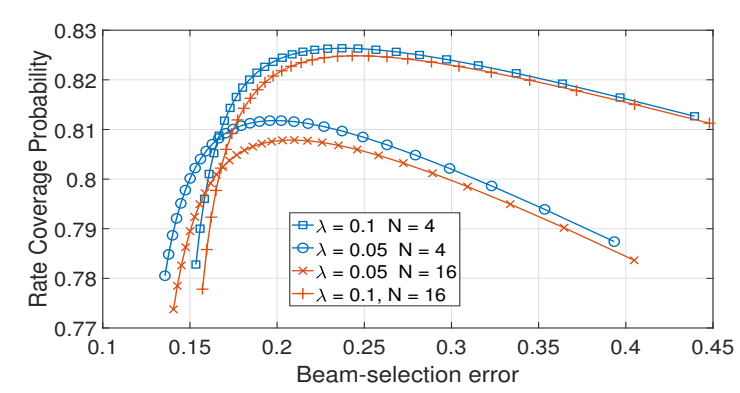

Figure 9. Rate coverage probability vs the beam-selection error.

probability gradually decreases with increasing $N$ until a certain value beyond which the beam becomes so thin that the misalignment error increases.

\section{Localization Data-Rate Trade-off}

In Fig. 9 we plot the rate coverage probability of the typical user with respect to the average beam-selection error, $\overline{\mathcal{P}}_{B S}$ for different beam dictionary sizes. We tune the value of $\overline{\mathcal{P}}_{B S}$ by modifying the value of $\beta$. We observe that for all beam dictionary sizes, as the value of $\overline{\mathcal{P}}_{B S}$ increases (i.e., as the localization estimation performance degrades), the rate coverage probability is initially improved. Thereafter, it reaches an optimal value for a certain $\overline{\mathcal{P}}_{B S}$ and decreases on further increasing the value of $\overline{\mathcal{P}}_{B S}$. This highlights the non-trivial trade-off between the localization and the data-rate performance in our system. This is all the more complex as the optimal value of $\beta$ (and hence the rate coverage probability) depends on both the BS deployment density and the dictionary size. To achieve very low values of $\overline{\mathcal{P}}_{B S}$, sufficient resources need to be allotted for the localization phase thus leading to efficient beam-selection and beam-alignment. A small increase in the value of $\overline{\mathcal{P}}_{B S}$ does not result in a large degradation of the localization performance but, in contrast, enhances the datarate as more resources are assigned to the data-communication phase. However, further increasing the value of $\overline{\mathcal{P}}_{B S}$ after a certain $\beta$ (i.e., $\beta^{*}$ ) deteriorates the rate coverage. This is because poor localization leads to a high beam selection errors. As a result, the effective antenna gains at the transmitter and receiver sides decrease, which directly reduces the useful received signal power, while the interference power remains same. Overall, this leads to limited rate performance. Another interesting observation in this figure is that in order to achieve the same coverage performance, the beam-selection error is slightly larger in case of larger beam-dictionaries. This is due to the thinner beams in larger beam-dictionaries, which increase the probabilities that the users lie outside the serving beam.

\section{Rate Coverage Performance and Trends}

In Figs. 10a and 10b we plot the rate coverage probability with respect to the resource partitioning factor $\beta$ varying the antenna gain parameter $G_{0}$ and the BS deployment density. First, we note again that there exists an optimal $\beta^{*}$ for each beam dictionary size, for which the rate coverage probability is maximized. More interestingly, the value of $\beta^{*}$ is not unique and is dependent not only on the dictionary size but 


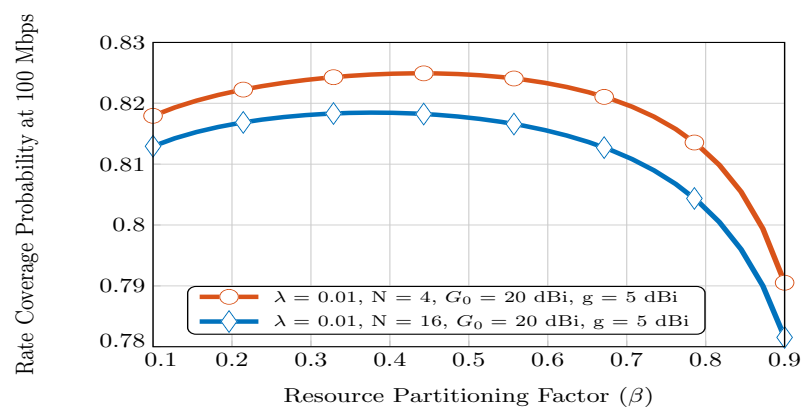

(a)

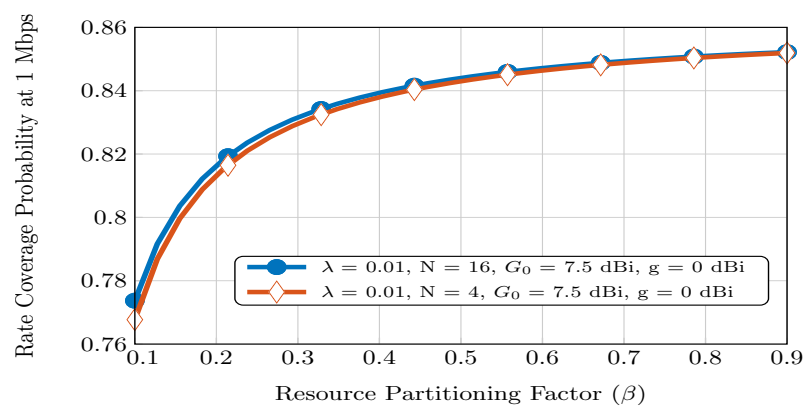

(b)

Figure 10. Rate coverage probability versus the resource partitioning factor for different beam dictionary sizes.

also on the system parameters such as antenna gains. From Fig. 10a we can see that the optimum value of $\beta$ decreases for higher $N$, i.e., thinner beamwidth. This is because with thinner beamwidth, the localization resources should be increased to limit the probability that the UE lies outside the coverage area of the beam.

When the antenna gain is smaller $\left(G_{0}=7.5 \mathrm{dBi}\right)$, we see in Fig. 10b that the rate coverage (at 1 Mbps contrary to 100 Mbps as before) increases with $\beta$. With $G_{0}=7.5 \mathrm{dBi}$, the positioning accuracy is limited (for any value of $\beta$ ), while increasing $\beta$ simply increases the communication resources, thereby augmenting the coverage. In this case, a smaller beamwidth (with $N=16$ ) provides slightly better coverage than a larger beamwidth (with $N=4$ ), since with limited localization accuracy, the rate coverage simply increases with decreasing $\theta$ due to higher radiated power. It must be noted that the rate coverage performance does not only depend on the antenna gains and $\lambda$, but also on the measurement noise. We study this point in the following sub-section.

\section{E. Optimal Partition factor and Beam Dictionary Size}

In this Section, we discuss the results obtained solving the transmit beamwidth and radio frame structure problem presented in (23). In Fig. 11a we plot the optimal values of $\beta$ with respect to the BS deployment density $\lambda$ and the noise power $N_{0} B$ [dBW]. For the optimization problem, we have considered $\epsilon=\epsilon^{\prime}=0.1$. With low noise power (e.g., $-50 \mathrm{dBW}$ ) the optimal value of $\beta$ is closer to 1 for higher $\lambda$. This is due to the fact that for low estimation noise and densely deployed BSs, even a limited amount of resources allocated to the localization phase results in a good localization performance. Thus, the optimal solution is to allocate large

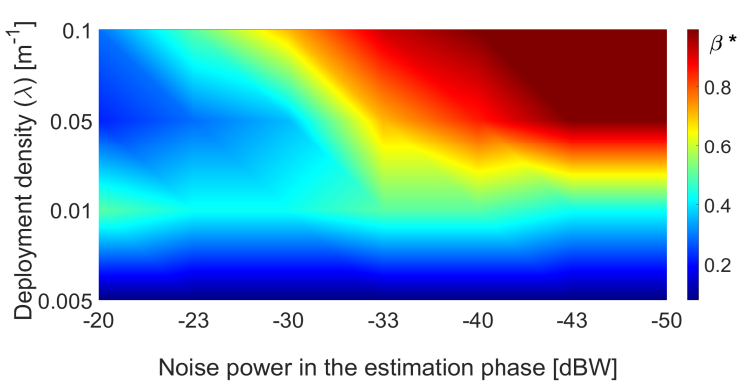

(a)

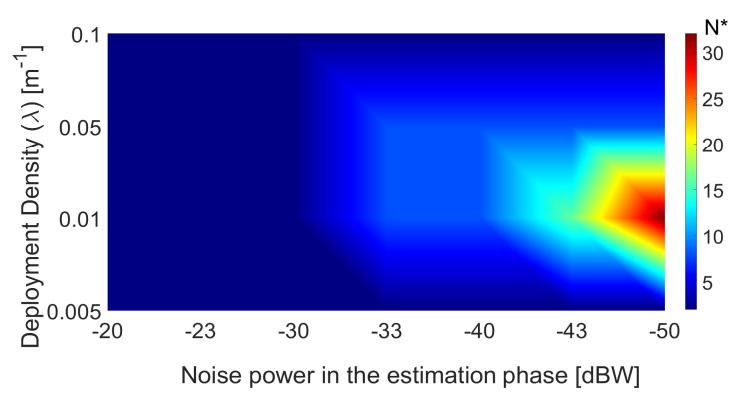

(b)

Figure 11. (a) Optimal value of $\beta$ with respect to deployment density and noise; (b) Optimal beam-dictionary size with respect to deployment density and the noise.

resources to the data phase for enhancing the rate coverage. On the other hand, for sparsely deployed BSs, larger amount of resources are required for efficient localization and the value of $\beta$ decreases, even for the case of low estimation noise.

Interestingly, in the case of high estimation noise (e.g., $N_{0} B=-20 \mathrm{dBW}$ ), when increasing the small cell density, the optimal $\beta$ increases at first and then decreases. This is due to the fact that for dense deployment of BSs, in case of high noise power of estimation, the effect of the beam-selection error is notable due to the concurrent large interference (since interfering BSs are closer due to higher density). This requires a lower value of optimal $\beta$ to facilitate efficient localization and reduce localization errors. Thus for higher estimation noise, the behaviour of optimal $\beta$ is not monotonous with respect to the deployment density.

In Fig. 11b we plot the optimal beam-dictionary size with respect to $\lambda$ and $N_{0} B$. For high estimation noise power, large beams (i.e., smaller dictionaries) must be used so as that the beam-selection error is limited. In case the estimation noise is low (e.g. $-50 \mathrm{dBW}$ ), the optimal size of the beam dictionary at first increases with the deployment density, due to the fact that larger antenna gains improve the rate coverage. However, after a certain point (i.e., for very dense deployments), the optimal beam-dictionary size decreases to limit the beamselection errors, which would have a large impact on the user performance due to the concurrent high interference.

\section{F. Impact of mobility of the users}

Finally, we note that the optimal beamwidth depends on the mobility of the users. Although, a comprehensive treatment of the user mobility, handover, and beam-switching mechanisms 


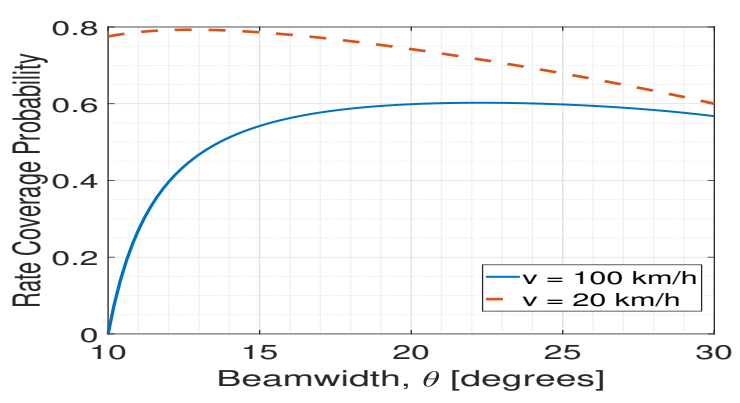

Figure 12. Rate coverage probability of a mobile user with respect to the serving beamwidth for different velocities of the user.

is beyond the scope of this paper, we present a preliminary numerical analysis to discuss some salient trends. In particular, we assume that the users in the network arrive at random locations, uniformly distributed within the coverage area of the typical BS, and are moving along the road with a uniform velocity $v$ [47]. In the initial access phase, a new user is allocated a serving beam and the BS does not employ any beam-switching mechanism. The users are assumed to request a data transfer of $b$ bits and in case the user moves out of the coverage area of the BS before the transfer of $b$ bits, we assume the user to be in outage. Naturally, this results in an interesting trade-off between the geographical coverage area and the radiated power with an increasing beamwidth. In Fig. 12 we plot the rate coverage probability of the mobile user with respect to the BS beamwidth for two different values of user velocity. Regardless of the velocity, the rate coverage first increases with beamwidth so as to increase the effective time the user spends in the main lobe of the BS. It reaches a peak and then starts to decrease since for large values of beamwidth the radiated power decreases. Naturally, a larger velocity results in a lower coverage probability. Furthermore, for a larger velocity, the optimal value of $\theta$ is larger as compared to the case with lower velocity. This is due to the fact that a smaller value of beamwidth results in a lower time spend by the user within the allotted beam. This indicates that for catering to users moving with a higher speed, the operator must necessarily increase the optimal $\theta$.

In practical systems, the operator should employ suitable mechanisms to adapt the serving beamwidth according to the velocity of the users. In particular, for highway scenarios, the beamwidth can be tuned according to the speed limits. Whereas, for urban scenarios, lookup tables can be maintained to map appropriate beamwidth for a set of user velocities.

\section{CONCLUSION}

In this paper we studied a mm-wave system deployed along the roads of a city to support localization and communication services simultaneously. We have proposed a novel localization bound-assisted initial beam-selection method for the mobile users, which reduces the latency of initial access by upto $75 \%$. Then the localization performance bounds have also been used to derive the downlink data-rate of the network in a system supporting jointly the localization and communication services. Our results highlight that increasing the resources allocated to the localization functions may or may not enhance the user data-rate. As a result, the study of the optimal resource partitioning factor is non-trivial. Consequently, we have highlighted and explored the main trends in the optimal resource partitioning factor and mm-wave beamwidth with respect to the rate coverage probability, with varying BS deployment density, antenna gain, and estimation noise. Finally, we provided several key system-design insights and guidelines based on our results. This will aid a network operator to cater to the outdoor mobile users, which are a key target for the first generation deployments of outdoor mm-wave BS.

\section{APPENDIX A}

Proof of Lemma 2: Beam-selection error occurs for a user in coverage of the beam $j$ of beamwidth $\theta_{k}$ when the estimated position lies outside the beam $j$. Thus, the probability of beamselection error, in case the user is estimated to be located at $\hat{d}$, is computed as:

$$
\begin{aligned}
\mathcal{P}_{B S, j, k}\left(d, \sigma_{d}^{2}\right) & =\mathbb{P}\left(\hat{d}<d_{L_{j N}}\right)+\mathbb{P}\left(\hat{d}>d_{R_{j N}}\right) \\
& \stackrel{(a)}{=} 1-\mathcal{Q}\left(\frac{d_{L_{j N}}-d}{\sigma_{d}}\right)+\mathcal{Q}\left(\frac{d_{R_{j N}}-d}{\sigma_{d}}\right),
\end{aligned}
$$

where (a) is due to the Gaussian nature of the error around mean $d$ and variance $\sigma_{d}^{2}$. Then, the probability of beamselection error and the typical user is in the coverage area of the $j$-th beam:

$$
\overline{\mathcal{P}}_{B S, j, k}=\int_{d_{L_{j N}}}^{d_{R_{j N}}} \mathcal{P}_{B S, j, N}(x) f_{d}(x) d x .
$$

Finally, the average beam-selection error for the localization based beam-selection scheme with a beam-dictionary size of $N$ is calculated as: $\overline{\mathcal{P}}_{B S}=\mathbb{E}_{d_{a}}\left[\sum_{j=1}^{N\left(d_{a}\right)} \overline{\mathcal{P}}_{B S, j, k}\right]$.

\section{APPENDIX B}

Proof of Theorem 1: Let index 1 denote the serving BS and $z_{k}=\sqrt{d_{k}^{2}+h_{B}^{2}}$ the distance between the $k$-th BS and the typical UE. The probability that the SINR at the typical user is larger than a threshold $T$, in case of absence of beamselection error and misalignment error is:

$$
\begin{aligned}
& \mathcal{T}_{0}=\mathbb{P}\left(S I N R_{C} \geq T\right) \\
& =\mathbb{P}\left(\frac{P_{t} K \Gamma z_{1}^{-\alpha_{L}}\left|f_{1}\right|^{2}}{N_{0}+P_{t} K g^{2}\left(\sum_{i \in \xi_{L} \backslash\{1\}} z_{i}^{-\alpha_{L}}\left|f_{i}\right|^{2}+\sum_{j \in \xi_{N}} z_{j}^{-\alpha_{N}}\left|f_{j}\right|^{2}\right)} \geq T\right) \\
& =\mathbb{P}\left(\left|f_{1}\right|^{2} \geq \frac{T N_{0}+P_{t} K g^{2}\left(\sum_{i \in \xi_{L} \backslash\{1\}} z_{i}^{-\alpha_{L}}\left|f_{i}\right|^{2}+\sum_{j \in \xi_{N}} z_{j}^{-\alpha_{N}}\left|f_{j}\right|^{2}\right)}{P_{t} K \Gamma z_{1}^{-\alpha_{L}}}\right) \\
& =\sum_{n=1}^{N_{L}}(-1)^{n+1}\left(\begin{array}{c}
N_{L} \\
n
\end{array}\right) \\
& \mathbb{E}\left[\operatorname { e x p } \left(-\frac{n \eta_{L} T N_{0}}{P_{t} K \Gamma z_{1}-\alpha_{L}}-\frac{n \eta_{L} T g^{2} \sum_{i \in \xi_{L} \backslash\{1\}} z_{i}^{-\alpha_{L}}\left|f_{i}\right|^{2}}{\Gamma z_{1}^{-\alpha_{L}}}-\right.\right. \\
& \left.\left.\frac{n \eta_{L} T g^{2} \sum_{j \in \xi_{N}} z_{j}^{-\alpha_{N}}\left|f_{j}\right|^{2}}{\Gamma z_{1}^{-\alpha_{L}}}\right)\right] \\
& =\sum_{n=1}^{N_{L}}(-1)^{n+1}\left(\begin{array}{c}
N_{L} \\
n
\end{array}\right) \exp \left(-\frac{n \eta_{L} T N_{0}}{P_{t} K \Gamma z_{1}^{-\alpha_{L}}}\right) \\
& \mathbb{E}_{\left|f_{i}\right|^{2}, \xi_{L} \backslash\{1\}}\left[\exp \left(-\frac{n \eta_{L} T g^{2} \sum_{i \in \xi_{L} \backslash\{1\}} z_{i}^{-\alpha_{L}}\left|f_{i}\right|^{2}}{\Gamma z_{1}^{-\alpha_{L}}}\right)\right]
\end{aligned}
$$


$\mathbb{E}_{\left|f_{j}\right|^{2}, \xi_{N}}\left[\exp \left(-\frac{n \eta_{L} T g^{2} \sum_{j \in \xi_{N}} z_{j}^{-\alpha_{N}}\left|f_{j}\right|^{2}}{\Gamma z_{1}^{-\alpha_{L}}}\right)\right]$,

where $\Gamma=\gamma_{B}\left(\theta_{B}\right) \gamma_{U}\left(\theta_{U}\right)$ and $\eta_{L}=N_{L}\left(N_{L} !\right)^{-\frac{1}{N_{L}}}[10]$. Now,

$\mathbb{E}_{\left|f_{i}\right|^{2}, \xi_{L} \backslash\{1\}}\left[\exp \left(-\frac{n \eta_{L} T g^{2} \sum_{i \in \xi_{L} \backslash\{1\}} z_{i}^{-\alpha_{L}}\left|f_{i}\right|^{2}}{\Gamma z_{1}^{-\alpha_{L}}}\right)\right]$
$=\mathbb{E}\left[\prod_{i \in \xi_{L} \backslash\{1\}} \mathbb{E}_{\left|f_{i}\right|^{2}}\left[\exp \left(-\frac{n \eta_{L} T g^{2} z_{i}^{-\alpha_{L}}\left|f_{i}\right|^{2}}{\Gamma z_{1}^{-\alpha_{L}}}\right)\right]\right]$
$=\exp \left(\int_{d_{1}}^{d_{S}} 1-\mathbb{E}_{\left|f_{i}\right|^{2}}\left[\exp \left(-\frac{n \eta_{L} T g^{2}\left(x^{2}+h_{B}^{2}\right)^{\frac{-\alpha_{L}}{2}}\left|f_{i}\right|^{2}}{\Gamma z_{1}^{-\alpha_{L}}}\right)\right]\right.$

$2 \lambda x d x)$

$=\exp \left(-2 \lambda \int_{d_{1}}^{d_{S}} 1-\frac{1}{\left(\frac{\eta_{L} T g^{2}\left(x^{2}+h_{B}^{2}\right)^{\frac{-\alpha}{2}}}{\left.N_{L} \Gamma z_{1}^{-\alpha} f_{i}\right|^{2}}\right)^{N_{L}}} x d x\right)$.

The NLOS case follows similarly. To calculate $\mathcal{T}_{B S}$ and $\mathcal{T}_{M A}$, respectively in the events of beam-selection error and misalignment, we replace the values of $\gamma_{B}\left(\theta_{B}\right)$ and $\gamma_{U}\left(\theta_{U}\right)$ with $g$ according to (3). Then, from the theorem of total probability, the SINR coverage at a distance $d_{1}$ is calculated. Conditioning on $d_{1}$ lying between $d_{L_{i}}$ and $d_{R_{i}}$ completes the proof.

\section{REFERENCES}

[1] A. De Domenico et al., "Making 5G Millimeter-Wave Communications a Reality," IEEE Wireless Communications, vol. 24, no. 4, pp. 4-9, Aug. 2017.

[2] 3GPP TSG RAN, "TR 38.900, Study on channel model for frequency spectrum above $6 \mathrm{GHz}, "$ v14.1.0, Sept. 2016.

[3] T. L. Marzetta et al., "Massive MIMO for Next Generation Wireless Systems," IEEE Commun. Mag., vol. 52, no. 2, pp. 186-195, Feb. 2014.

[4] T. S. Rappaport et al., "Millimeter Wave Mobile Communications for 5G Cellular: It Will Work!" IEEE Access, vol. 1, pp. 335-349, 2013.

[5] A. Ghosh et al., "Millimeter-Wave Enhanced Local Area Systems: A High-Data-Rate Approach for Future Wireless Networks," IEEE J. Sel. Areas Commun., vol. 32, no. 6, pp. 1152-1163, June 2014.

[6] G. Ghatak, A. De Domenico, and M. Coupechoux, "Coverage Analysis and Load Balancing in HetNets With Millimeter Wave Multi-RAT Small Cells," IEEE Trans. Wireless Commun., vol. 17, no. 5, pp. 3154-3169, May 2018.

[7] Y. Qi and M. Nekovee, "Coordinated Initial Access in Millimetre Wave Standalone Networks," in Proc. IEEE INFOCOM Workshops, Apr. 2016, pp. 59-64.

[8] N. Garcia et al., "Location-aided mm-Wave Channel Estimation for Vehicular Communication," in Proc. IEEE SPAWC, July 2016, pp. 1-5.

[9] G. Ghatak et al., "Positioning Data-Rate Trade-off in mm-Wave Small Cells and Service Differentiation for 5G Networks," in Proc. IEEE VTC Spring, June 2018, pp. 1-5.

[10] T. Bai and R. W. Heath, "Coverage and Rate Analysis for MillimeterWave Cellular Networks," IEEE Trans. Wireless Commun.,, vol. 14, no. 2, pp. 1100-1114, Feb. 2015.

[11] M. Di Renzo, "Stochastic Geometry Modeling and Analysis of MultiTier Millimeter Wave Cellular Networks," IEEE Trans. Wireless Commun., vol. 14, no. 9, pp. 5038-5057, Sept. 2015.

[12] H. Elshaer et al., "Downlink and Uplink Cell Association With Traditional Macrocells and Millimeter Wave Small Cells," IEEE Trans. Wireless Commun., vol. 15, no. 9, pp. 6244-6258, Sept. 2016.

[13] Y. Li et al., "Design and Analysis of Initial Access in Millimeter Wave Cellular Networks," IEEE Trans. Wireless Commun., vol. 16, no. 10, pp. 6409-6425, Oct. 2017.

[14] Y. Yang et al., "Fast and Reliable Initial Access with Random Beamforming for mmWave Networks," arXiv preprint arXiv:1812.00819, 2018.
[15] F. Lemic et al., "Localization as a Feature of mmWave Communication," in Proc. IWCMC, Sept. 2016, pp. 1033-1038.

[16] A. Shahmansoori et al., "5G Position and Orientation Estimation through Millimeter Wave MIMO," in Proc. IEEE Globecom Workshops, Dec. 2015.

[17] - , "Position and Orientation Estimation Through Millimeter-Wave MIMO in 5G Systems," IEEE Trans. Wireless Commun., vol. 17, no. 3 , pp. 1822-1835, Mar. 2018.

[18] R. Koirala et al., "Localization bound based beamforming optimization for multicarrier mmwave MIMO," in Proc. WPNC, Oct. 2017, pp. 1-6.

[19] — "Localization optimal multi-user beamforming with multi-carrier mmwave MIMO," in Proc. IEEE PIMRC, Sept. 2018.

[20] A. Kakkavas et al., "5G downlink multi-beam signal design for LOS positioning," ArXiv, vol. abs/1906.01671, 2019.

[21] G. Destino and H. Wymeersch, "On the Trade-off between Positioning and Data Rate for mm-Wave Communication," in Proc. IEEE ICC Workshops, May 2017, pp. 797-802.

[22] D. Kumar et al., "On trade-off between 5G positioning and mmwave communication in a multi-user scenario," in 2018 8th International Conference on Localization and GNSS (ICL-GNSS), June 2018, pp. 1-5.

[23] H. L. Van Trees, Detection, Estimation, and Modulation Theory. John Wiley \& Sons, 2004

[24] F. Maschietti et al., "Robust location-aided beam alignment in millimeter wave massive mimo," in GLOBECOM 2017 - 2017 IEEE Global Communications Conference, Dec. 2017, pp. 1-6.

[25] O. Igbafe et al., "Location-aware beam alignment for mmwave communications," arXiv preprint arXiv:1907.02197, 2019.

[26] G. E. Garcia et al., "Transmitter beam selection in millimeter-wave mimo with in-band position-aiding," IEEE Transactions on Wireless Communications, vol. 17, no. 9, pp. 6082-6092, Sept. 2018.

[27] — "Fast in-band position-aided beam selection in millimeter-wave MIMO," IEEE Access, vol. 7, pp. 142 325-142 338, 2019.

[28] R. Koirala et al., "Throughput Characterization and Beamwidth Selection for Positioning-Assisted mmWave Service," in Proc. ACSSC, Oct. 2018, pp. 438-443.

[29] M. S. Bartlett, "Periodogram analysis and continuous spectra," Biometrika, vol. 37, no. 1/2, pp. 1-16, 1950.

[30] J. Capon, "High-resolution frequency-wavenumber spectrum analysis," Proc. IEEE, vol. 57, no. 8, pp. 1408-1418, Aug. 1969.

[31] R. O. Schmidt, "A signal subspace approach to multiple emitter location and spectral estimation." Ph.D. dissertation, Dept. Elect. Eng., Stanford Univ., Stanford, CA, USA, 1982.

[32] H. El-Sayed, G. Athanasiou, and C. Fischione, "Evaluation of localization methods in millimeter-wave wireless systems," in 2014 IEEE 19th International Workshop on Computer Aided Modeling and Design of Communication Links and Networks (CAMAD), 2014, pp. 345-349.

[33] "Samsung and Verizon announce first 5G customer trials set to begin in Q2 2017," 2017. [Online]. Available: https://news.samsung.com/global/ samsung-and-verizon-announce-first-5g-customer-trials- set-to-begin-in-q2-2017

[34] "AT\&T, San Jose Agree on New Wireless Infrastructure to Facilitate 5G," Apr 2018. [Online]. Available: https://www.radioworld.com/trends-1/ at-t-san-jose-agree-on-new-wireless-infrastructure-to-facilitate- $5 \mathrm{~g}$

[35] S. N. Chiu et al., Stochastic Geometry and its Applications. John Wiley \& Sons, 2013

[36] G. Ghatak, A. De Domenico, and M. Coupechoux, "Small cell deployment along roads: Coverage analysis and slice-aware rat selection," IEEE Transactions on Communications, 2019.

[37] A. M. Hunter, J. G. Andrews, and S. Weber, "Transmission Capacity of ad hoc Networks with Spatial Diversity," IEEE Trans. Wireless Commun., vol. 7, no. 12, pp. 5058-5071, Dec. 2008.

[38] H. Shokri-Ghadikolaei et al., "Millimeter Wave Cellular Networks: A MAC Layer Perspective," IEEE Trans. Wireless Commun., vol. 63, no. 10, pp. 3437-3458, Oct. 2015.

[39] J. G. Andrews et al., "Modeling and Analyzing Millimeter Wave Cellular Systems," IEEE Trans. Wireless Commun., vol. 65, no. 1, pp. 403-430, Jan. 2017.

[40] N. Garcia, H. Wymeersch, and D. T. M. Slock, "Optimal Precoders for Tracking the AoD and AoA of a mmWave Path," IEEE Trans. Signal Process., vol. 66, no. 21, pp. 5718-5729, Nov. 2018.

[41] J. Nikolić, Z. Perić, and A. Jovanović, "Novel approximations for the qfunction with application in sqnr calculation," Digital Signal Processing, vol. 65, pp. 71-80, 2017.

[42] Y. Chen and N. C. Beaulieu, "Solutions to infinite integrals of gaussian q-function products and some applications," IEEE communications letters, vol. 11, no. 11, pp. 853-855, 2007. 
[43] N. Michelusi and M. Hussain, "Optimal Beam Sweeping and Communication in Mobile Millimeter-Wave Networks," Proc. IEEE ICC, May 2018.

[44] T. Baykas et al., "IEEE 802.15. 3c: the first IEEE wireless standard for data rates over $1 \mathrm{~Gb} / \mathrm{s}$," IEEE Commun. Mag., vol. 49, no. 7, pp. 114-121, 2011.

[45] G. Anastasi et al., "IEEE 802.11 ad hoc Networks: Performance Measurements," in Proc. ICDCS, May 2003, pp. 758-763.

[46] M. Hussain and N. Michelusi, "Throughput Optimal Beam Alignment in Millimeter Wave Networks," in Proc. ITA Workshop, Feb. 2017, pp. $1-6$.

[47] P. Madadi, F. Baccelli, and G. de Veciana, "On temporal variations in mobile user snr with applications to perceived qos," in 2016 14th International Symposium on Modeling and Optimization in Mobile, Ad Hoc, and Wireless Networks (WiOpt), 2016, pp. 1-8. 